ZENS. IV. SIMILAR MORPHOLOGICAL CHANGES

ASSOCIATED WITH MASS QUENCHING AND

ENVIRONMENT QUENCHING AND THE RELATIVE

IMPORTANCE OF BULGE GROWTH VERSUS THE FADING

OF DISKS

Carollo, C. M.

2016-02-20

Carollo , C M , Cibinel , A , Lilly , S J , Pipino , A, Bonoli , S , Finoguenov , A, Miniati , F , Norberg , P \& Silverman , J D 2016 , ' ZENS. IV. SIMILAR MORPHOLOGICAL CHANGES ASSOCIATED WITH MASS QUENCHING AND ENVIRONMENT QUENCHING AND THE RELATIVE IMPORTANCE OF BULGE GROWTH VERSUS THE FADING OF DISKS ' , Astrophysical Journal , vol. 818 , no. 2 , 180 . https://doi.org/10.3847/0004-637X/818/2/180

http://hdl.handle.net/10138/183633

https://doi.org/10.3847/0004-637X/818/2/180

unspecified

publishedVersion

Downloaded from Helda, University of Helsinki institutional repository.

This is an electronic reprint of the original article.

This reprint may differ from the original in pagination and typographic detail.

Please cite the original version. 


\title{
ZENS. IV. SIMILAR MORPHOLOGICAL CHANGES ASSOCIATED WITH MASS QUENCHING AND ENVIRONMENT QUENCHING AND THE RELATIVE IMPORTANCE OF BULGE GROWTH VERSUS THE FADING OF DISKS*
}

\author{
C. M. Carollo ${ }^{1}$, A. Cibinel ${ }^{1,2}$, S. J. Lilly ${ }^{1}$, A. Pipino ${ }^{1}$, S. Bonoli ${ }^{3}$, A. Finoguenov ${ }^{4}$, \\ F. Miniati ${ }^{1}$, P. NorberG ${ }^{5}$, and J. D. Silverman ${ }^{6}$ \\ ${ }^{1}$ Institute for Astronomy, ETH Zurich, CH-8093 Zurich, Switzerland; marcella@phys.ethz.ch \\ 2 Astronomy Centre, Department of Physics and Astronomy, University of Sussex, Brighton, BN1 9QH, UK \\ ${ }^{3}$ Centro de Estudios de Fisica del Cosmos de Aragon, Spain \\ ${ }^{4}$ Department of Physics, University of Helsinki, FI-00014 Helsinki, Finland \\ ${ }^{5}$ Institute for Computational Cosmology, Department of Physics, Durham University, South Road, Durham DH1 3LE, UK \\ ${ }^{6}$ Kavli Institute for the Physics and Mathematics of the Universe (WPI), Todai Institutes for Advanced Study, The University of Tokyo, Kashiwa, 277-8583, Japan \\ Received 2014 February 6; accepted 2016 January 5; published 2016 February 18
}

\begin{abstract}
We use the low-redshift Zurich Environmental Study (ZENS) catalog to study the dependence of the quenched satellite fraction at $10^{10.0} M_{\odot} \rightarrow 10^{11.5} M_{\odot}$, and of the morphological mix of these quenched satellites, on three different environmental parameters: group halo mass, halo-centric distance, and large-scale structure (LSS) overdensity. Within the two mass bins into which we divide our galaxy sample, the fraction of quenched satellites is more or less independent of halo mass and the surrounding LSS overdensity, but it increases toward the centers of the halos, as found in previous studies. The morphological mix of these quenched satellites is, however, constant with radial position in the halo, indicating that the well-known morphology-density relation results from the increasing fraction of quenched galaxies toward the centers of halos. If the radial variation in the quenched fraction reflects the action of two quenching processes, one related to mass and the other to environment, then the constancy with radius of the morphological outcome suggests that both have the same effect on the morphologies of the galaxies. Alternatively, mass and environment quenching may be two reflections of a single physical mechanism. The quenched satellites have larger bulge-to-total ratios $(B / T)$ and smaller half-light radii than the starforming satellites. The bulges in quenched satellites have very similar luminosities and surface brightness profiles, and any mass growth of the bulges associated with quenching cannot greatly change these quantities. The differences in the light-defined $B / T$ and in the galaxy half-light radii are mostly due to differences in the disks, which have lower luminosities in the quenched galaxies. The difference in galaxy half-light radii between quenched and star-forming satellites is however larger than can be explained by uniformly fading the disks following quenching, and the quenched disks have smaller scale lengths than in star-forming satellites. This can be explained either by a differential fading of the disks with galaxy radius or the disks being generally smaller in the past, both of which would be expected in an inside-out disk growth scenario. The overall conclusion is that, at least at low redshifts, the structure of massive quenched satellites at these masses is produced by processes that operate before the quenching takes place. A comparison of our results with semianalytic models argues for a reduction in the efficiency of group halos in quenching their disk satellites and for mechanisms to increase the $B / T$ of low-mass quenched satellites.
\end{abstract}

Key words: galaxies: bulges - galaxies: evolution - galaxies: groups: general - galaxies: halos - galaxies: statistics - galaxies: structure

\section{INTRODUCTION}

The star-formation rate (SFR) and the morphology of a galaxy are key diagnostics of its evolutionary stage. These properties are broadly correlated, in the sense that galaxies with dominant disks tend to have higher specific SFR (sSFR $=\mathrm{SFR} / M_{\text {galaxy }}$, with $M_{\text {galaxy }}$ the galaxy stellar mass) than galaxies with a pronounced spheroidal component. There is solid observational evidence that the cessation of star formation in some galaxies, which results in the emergence of "quenched" passive galaxies, depends on both galaxy mass and environment (e.g., Dressler 1980; Balogh et al. 2004; Baldry et al. 2006; Kimm et al. 2009). In the Sloan Digital Sky-Survey (SDSS; York et al. 2000) and zCOSMOS (Lilly et al. 2007, 2009) samples, the effects of galaxy mass and environment in

\footnotetext{
* Based on observations collected at the European Southern Observatory, La Silla Chile. Program ID 177.A-0680.
}

the quenching of galaxies appear to be separable, both in the local universe and at least up to redshift $z=1$ (Peng et al. 2010; Kovač et al. 2014). In other words, it is possible to write the fraction of galaxies that survive as blue star-forming galaxies as the product of two functions, one a function of stellar mass only, and the other a function of "environment" only. Each of these functions is therefore the extra effect of mass, or environment, over the effects of the other. In these works, the environmental measure used is an Nth-nearestneighbor estimate of the local density of galaxies. This separability of the quenching effects of galaxy mass and environment has been taken to argue in favor of two independent physical processes, which Peng et al. (2010) called mass quenching (independent of environment) and environment quenching (independent of stellar mass), a terminology that we also adopt in this paper. These two quenching processes seem to be quite sharp, in the sense that the sSFRs of the surviving star-forming galaxies that are not 
quenched are almost constant with both galaxy stellar mass and environment (Peng et al. 2010, 2012).

The physical nature of mass and environment quenching is, however, unclear. Several theoretical suggestions have been advanced for what we here are calling mass quenching. Some invoke some form of halo physics (e.g., Birnboim et al. 2007; Hearin \& Watson 2013; Woo et al. 2013), and others include active galactic nucleus (AGN) feedback (see, e.g., Haas et al. 2013 and references therein). Other possibilities include so-called morphological quenching (Dekel \& Birnboim 2008; Martig et al. 2009, 2013; Genzel et al. 2014). Likewise, for environment quenching, many physical processes have been proposed, including ram pressure stripping, accretion shocks, and removal of hot gas (Birnboim \& Dekel 2003; Kawata \& Mulchaey 2008; Cen 2014), with most explanations generally invoking some form of gas removal from galaxies as they fall into a group potential (e.g., Feldmann et al. 2011; De Lucia et al. 2012 and references therein). An interesting question is whether satellite-satellite mergers in low-mass halos also play a role as a channel for environmental quenching (see, e.g., Pipino et al. 2014, Paper V in our Zurich Environmental Study - hereafter ZENS-series).

The difficulty in identifying the mechanisms that are acting in both quenching mechanisms is at least in part due to both real and spurious correlations between different definitions of mass and environment that blur our understanding of "which mass" and "which environment" are the most relevant for galaxy evolution. Galaxy stellar mass is directly related to dark matter halo mass, at least for central galaxies. A wide range of environmental measures on different scales may be correlated with each other, from the global potential of the group dark matter halos, quantified by the halo mass, to the position and thus local density within the halos, to the density provided by the large-scale structure cosmic web (e.g., van den Bosch et al. 2008; Kimm et al. 2009; Hoyle et al. 2012; Peng et al. 2012; Woo et al. 2013; see also Carollo et al. 2013b, hereafter Paper I).

One fact that has become evident, however, is that the rank of a galaxy within the group dark matter halo is very important, that is, whether the galaxy is the first-ranked central galaxy, lying at the minimum of the potential, or a satellite galaxy orbiting within the group potential (e.g., Weinmann et al. 2006, 2009). As earlier also explored by van den Bosch et al. (2008); Peng et al. (2012) show that the environmental effects in their 2010 analysis of the SDSS are due to changes in the satellite population alone: their "environment quenching" is actually a "satellite quenching" process. Within this framework, mass quenching would be envisioned to act on all galaxies and be related either directly or indirectly to the stellar mass of the galaxy, whereas environment (i.e., satellite) quenching would act only on the satellites. Since the characteristic $M *$ of centrals and satellites is observed to be very similar, satellites must have experienced the same risk of mass quenching as central galaxies. It is an open question, however, whether this risk occurred when the satellites were actually centrals of their own halos, before they became satellites of a larger halo.

The same authors further showed that the fraction of satellite galaxies with red colors correlates much better with the local overdensity, as estimated with a fifth-nearest-neighbor parameter, loosely interpreted to be a measure of location within the groups, than with the optical richness of the group, which is a proxy for the dark matter halo mass (see also Paper I). Woo et al. (2013) also found that the fraction of quenched galaxies at fixed galaxy stellar mass shows a dependence on halo-centric distance. These authors furthermore reported that the quenching of satellite galaxies depends also on galaxy stellar mass, but only at large (and not at small) halo-centric distances, which they interpret to arise from a quenching dependence on subhalo mass for satellites that are recently accreted by a more massive halo. This could be rephrased as the increasing importance of mass-dependent mass quenching (affecting all galaxies) relative to mass-independent satellite quenching at low densities.

Important constraints for establishing the physical mechanisms behind mass quenching and satellite quenching can be obtained by studying the morphologies that are associated with different populations of quenched galaxies. It has become clear in the last few years that, in addition to the well-known connection between a quenched spectrum and an elliptical morphology, galaxies with a predominant disk component can also be quenched systems (e.g., Bundy et al. 2010; Kovač et al. 2010; Oesch et al. 2010). This prompts the question as to whether there is a dependence with environment in the morphological mix of the quenched galaxy population, and especially in the quenched satellite population. The quantification of any such dependence may help elucidate in which environments quenching is or is not associated with a morphological transformation and, more generally, which morphologies or morphological transformations can be associated with either mass or environment quenching (or both).

In this fourth paper in the ZENS series, we use the ZENS catalog of galactic and environmental properties published in Paper I to directly address this question by analyzing the morphologies of the quenched satellite populations that reside in a set of nearby galaxy groups that span a range of halo masses and surrounding large-scale structure (LSS) densities.

In ZENS Paper I, we derived three measures of environment: the LSS overdensity $\delta_{\mathrm{LSS}}$, the mass of the host group halo $M_{\mathrm{GROUP}}$, which we will here rename as $M_{\mathrm{Halo}}$, and the projected halo-centric distance $R$ relative to the characteristic radius of the group halo $R_{200}=\left(\frac{G M_{\text {Halo }}}{[10 H(z)]^{2}}\right)^{1 / 3}$, which we denote $R_{\text {vir }}$ (with $H(z)=H_{0} \sqrt{\Omega_{M}(1+z)^{3}+\Omega_{\Lambda}}$ the Hubble constant at the given redshift).

A strength of ZENS is to eliminate sample-dependent biases by using exactly the same sample of galaxies to perform such environment-versus-environment comparisons. These galaxies are 1455 members of 141 groups that were extracted from the Percolation-Inferred Galaxy Group, 2PIGG, catalog (Eke et al. 2004) of the 2dFGRS (Colless et al. 2001).

The present paper explores specifically, in fixed-mass bins, (1) how the fraction of quenched satellites depends on group halo mass, on halo-centric distance, and on LSS density; (2) how the morphological composition of the quenched satellite populations depend on these different environmental measures; and (3) how the structural properties of quenched satellites compare to similar properties of star-forming satellites of similar mass. Indirect estimates for the quenched fraction of the central galaxies of similar stellar mass are also discussed, in order to obtain a benchmark estimate for the relative contribution of mass quenching and satellite quenching at a given galaxy mass scale.

The paper is organized as follows. In Section 2 we briefly describe ZENS, and in particular the environmental parameters derived in Paper I as well as the morphological and spectral 
parameters for the ZENS galaxies that are derived respectively in Cibinel et al. (2013a), hereafter Paper II, and Cibinel et al. (2013b), hereafter Paper III. $^{7}$ In the Appendix we show the impact of including unrelaxed groups in the present analysis and define the ZENS sample that we use in the present study, which includes both relaxed and unrelaxed groups (see Section 2.1.1 for the definitions). In Section 3, we present the dependence on $M_{\mathrm{Halo}}$ and $R / R_{\mathrm{vir}}$ of the total fraction of quenched satellites $f_{Q}$, and of the fraction $f_{Q_{\mathrm{ETG}}}$ of quenched satellites that have an early-type galaxy (ETG) morphology, relative to the total quenched satellite population. In Section 4, we separately report the analysis of the satellite-quenched fraction and its morphological composition as a function of LSS density. In Section 5 we investigate the global structural properties of quenched and star-forming satellites and of their bulges and disks. Here we also discuss our results first in terms of the apparent morphological changes associated with mass and environment quenching, and second in comparison with traditional semianalytic models (SAMs) of galaxy formation. Section 6 summarizes our findings and highlights some open questions that emerge from our analysis.

All of our ZENS work is based on a $\Omega_{m}=0.3, \Omega_{\Lambda}=0.7$, and $h=0.7$ cosmological model.

\section{ESSENTIALS OF ZENS}

Details on the ZENS sample, multi-band data, environmental measurements, and measurements of galaxy morphology, structure, stellar mass, and SFR have been described in Papers I, II, and III. Briefly, the ZENS group sample spans the halo mass range from $\sim 10^{12.2} M_{\odot}$ to $\sim 10^{14.9} M_{\odot}$; this corresponds to a stellar content within galaxies between $9 \times 10^{9} M_{\odot}$ and $4 \times 10^{12} M_{\odot}$ (see Figure 11 of Paper I). Halo masses were determined from the group luminosities. SAMs were used to estimate the intrinsic uncertainty in the conversion between halo luminous and dark masses, and tests were performed to assess other systematic uncertainties, in particular the impact of interlopers and missed galaxies. Due to these uncertainties, the scattering of groups between the two halo mass bins, that is, cross talk between halo mass bins, may weaken any intrinsic dependence of galaxy properties on halo masses, a factor that we will take into account when analyzing our results. The ZENS group sample was selected to be in the narrow redshift range $0.05<z<0.0585$ and to include groups with at least five spectroscopically confirmed member galaxies down to the limiting magnitude $\left(b_{J}=19.45 \mathrm{mag}\right)$ of the 2dFGRS. We highlight below those aspects of ZENS that are most relevant to the current analysis.

\subsection{Definition of the Different Environments and Minimization of Environmental Cross Talk}

In computing different environmental parameters, which in principle should probe the surroundings of galaxies on different scales, several factors can introduce cross talk among such parameters. This complicates attempts to identify the physical mechanisms behind environmentally driven galaxy evolution. We discuss below some of the most important of these factors that are relevant for our analysis, and we summarize our approaches to minimizing their impact on the results.

\footnotetext{
7 The full ZENS catalog of galaxy and environmental measurements is published with Paper I.
}

\subsubsection{Identification of Centrals and Satellites and Definition of Relaxed and Unrelaxed Groups}

Accurate knowledge of group centers is crucial for reliably measuring variations of galaxy properties with halo-centric distances and differentiating central galaxies from satellite galaxies. Identifying the central galaxies and thus the group centers can, however, be difficult, especially for groups with few spectroscopically confirmed galaxy members. In optically selected galaxy group catalogs, the customary approach to identifying the central galaxy is to find the most massive, or sometimes even just the brightest, galaxy among those that are linked as a single group by a friends-of-friends algorithm. This leads in some cases to groups in which the identified central galaxy is substantially displaced from the group centers, either in projected distance or in velocity space.

To address this issue, in ZENS we classified groups as "relaxed" and "unrelaxed," depending on whether we could identify a single central galaxy that simultaneously satisfies the mass, halo-centric distance, and velocity requirements for it to be assigned the rank of central galaxy. Specifically, we initially identified the apparently most massive galaxy (i.e., the member galaxy with the highest best-fit stellar mass, as established from synthetic galaxy template fitting to the observed near-ultraviolet to near-infrared photometric spectral energy distributions (SEDs)). Groups were classified as relaxed if this most massive galaxy was located (1) within a radial distance of $0.5 R_{\mathrm{vir}}$ from the mass-weighted geometric center of the group and (2) within the central 68th percentile of the velocity distribution of the group. Groups in which the highest mass galaxy did not satisfy these space and velocity criteria were further studied to assess whether, within the errors of our galaxy stellar mass estimates, there was a different galaxy that satisfied all the abovementioned criteria within the errors. Groups for which this procedure found an alternative bona fide central galaxy were also classified as relaxed (but are excluded from the "clean" sample of relaxed groups to which we refer below). Groups in which no self-consistent solution could be found for a bona fide central, that is, a galaxy that, within the errors, satisfied all the mass, location, and velocity criteria, were classified as unrelaxed. The ZENS sample contains 59 unrelaxed groups and 82 relaxed groups (73 of which compose the "clean" sample of relaxed groups in which the central galaxy is the nominally most massive galaxy).

\subsubsection{An LSS Density Estimate Unaffected by Group Richness}

As pointed out by several authors, computations of the LSS density based on Nth-nearest-galaxy approaches correspond to different environments in halos of richness above or below the chosen " $N$," describing respectively inter- and intrahalo densities (Paper I, and, e.g., Peng et al. 2010; Woo et al. 2013). In ZENS we adopted a parameterization of the LSS density that does not switch meaning for galaxies in groups of richness above and below a given value and selfconsistently measures the intergroup density of the filamentary cosmic structure. Specifically, we traced the LSS density using a fifth-nearest-neighbor approach, adopting, however, the mass-weighted groups rather than their member galaxies as the tracers of the density field. All groups in the 2PIGG catalog within the redshift interval $d z= \pm 0.01$ of the group of interest were used in this computation, including "one-galaxy groups", namely isolated galaxies that are more luminous than a 
$z=0.07, b_{j}=19.1 \mathrm{mag}$ galaxy (corresponding to a median stellar mass of $\sim 10^{9.2} M_{\odot}$ as derived from the $b_{j}$ versus $M_{\text {galaxy }}$ relation for the ZENS galaxies). Our LSS density parameter thus reflects the location of a group in the cosmic web and not at all the local density that a given satellite will experience within its group halo since, by definition, all galaxy members of a given group are assigned an identical LSS density parameter.

\subsubsection{Disentangling Halo Mass and LSS Density Effects}

The physical association of high-mass halos with high LSS densities introduces a degeneracy that makes it difficult to disentangle the separate effects of physics within virialized massive halos (as parameterized by the halo mass) and dense regions of the cosmic web (as parameterized by the interhalo LSS density). To attempt to establish whether possible environmental trends are driven by one or the other of these physically distinct environments, we apply a high halo mass cutoff in the group sample when investigating possible effects of the LSS density on galaxy properties. Specifically, we use only groups with $M_{\text {Halo }} \leqslant 10^{13.7} M_{\odot}$ in Section 4 to study whether the surrounding LSS density affects either the quenched satellite fraction or its morphological composition. These halos of low to intermediate mass can be found over a wide range of LSS densities (see Paper I).

\subsection{Identification of the Quenched Galaxies}

To separate galaxies into quenched and (moderately or strongly) star-forming systems, we adopted a combination of several indicators. Quenched galaxies are required to satisfy all of the following spectral and color criteria: (1) no detected emission in $\mathrm{H} \alpha$ and $\mathrm{H} \beta$ and (2) $(\mathrm{NUV}-I)>4.8$, $(\mathrm{NUV}-B)>3.5$, and $(B-I)>1.2$.

The final assignment of a galaxy to the quenched population was based on the SSFR derived from an SED fit to its photometric data (see Section 2.3), validated, however, through the spectral and color requirements listed above. This multipleconstraint approach to the identification of quenched galaxies leads to a higher purity of the sample compared to the use of a single (often optical) color criterion; samples based on the latter may suffer from a nonnegligible contamination from, for example, dust-reddened star-forming galaxies, especially at masses $\lesssim 10^{11} M_{\odot}$ (see Paper III and Woo et al. 2013).

In discussing our results in Section 5, we will compare the bulge and disk properties of the quenched satellites with those of star-forming satellites of similar stellar mass in the ZENS sample. In our work we define galaxies to be strongly starforming if they show (1) strong [O II], [N II], $\mathrm{H} \alpha$, and $\mathrm{H} \beta$ line emission and (2) $(\mathrm{NUV}-I)<3.2$, $(\mathrm{NUV}-B)<2.3$, and $(B-I)<1.0$ colors. Galaxies with color and spectral properties between strongly star-forming and quenched galaxies are classified as moderately star-forming objects. Further details are given in Paper III.

\subsection{Estimates of Galaxy Stellar Masses and SFRs}

Galaxy stellar masses and SFRs were derived in Paper III by fitting model SEDs to the photometric data of the ZENS galaxies using the Zurich Extragalactic Bayesian Redshift Analyzer+ (ZEBRA+; Oesch et al. 2010). ZEBRA+ is an upgraded version of our publicly released ZEBRA code (Feldmann et al. 2006). Stellar population models were adopted from the Bruzual \& Charlot (2003) library with a Chabrier (2003a) initial mass function. We used two types of starformation histories: exponentially decaying models with a broad range of characteristic $\tau$ timescales (from very short to very long e-folding $\tau$ values) and constant star-formation models. Details on the grid of SED models are given in Table 2 of Paper III.

All SED fits were visually inspected to ensure consistency between the spectral+color analysis and the SFR estimates derived from the fits. For $2 \%$ of ZENS galaxies, a second iteration in the fits was required to resolve initial discrepancies between the SED-based SFRs and the spectral+color assessments. Specifically, the original SED fits to this small fraction of galaxies with clearly star-forming spectra and colors returned very low SFRs. In these few cases, we rerun the SED fits after restricting the sample of templates to starforming SED models only.

We use as our definition of stellar mass the integral of the past SFR. Our stellar masses are thus about 0.2 dex higher than the "actual" stellar mass that is sometimes used, that is, the stellar mass that remains after mass return to the interstellar medium from the evolving stellar population.

With our definition, the stellar mass completeness of the ZENS galaxy sample for quenched systems is $M_{\text {galaxy }} \sim 10^{10} M_{\odot}$.

\subsection{Galaxy Morphologies and B/T Decompositions}

A strength of ZENS is its correction for measurement biases in the structural parameters. These were calibrated to eliminate spurious trends with the size of the seeing-driven point-spread function (PSF) and with galaxy magnitude, size, concentration, and ellipticity. A quantitative structural-morphological classification was then implemented on the basis of these corrected structural parameters. The classification was mainly based on the light-defined bulge-to-total $(B / T)$ ratio, which was measured via two-dimensional bulge+disk fits to the galaxy surface brightness distributions, reinforced by imposing quantitative boundaries for the different morphological types in standard nonparametric quantities such as concentration, Gini, $M_{20}$, and smoothness parameters (see Table 2 and Figure 18 of Paper II for details). Bulges were modeled with a Sérsic profile and disks with a single exponential profile (see Paper II for details).

We note that in the literature the word "morphology" is typically associated with visual (or visual-like) classifications reflecting the combination of galaxy structure and starformation properties and involving no quantitative estimate of the contribution of the disk and bulge components (see, e.g., Conselice 2003; Lotz et al. 2004; van der Wel 2008; Nair \& Abraham 2010; Kartaltepe et al. 2015 or the Galaxy Zoo project, Lintott et al. 2011). From a physical perspective, however, it is the $B / T$ ratio that carries most of the meaning of a "morphological class." In our ZENS work, we have therefore chosen to avoid the subjectivity of a visual morphological classification and adopted a physically meaningful definition of "morphology" that is primarily based on the $B / T$ ratio. We furthermore make use of nonparametric quantities to facilitate the separation between neighboring morphological classes (e.g., S0 and early-type spiral galaxies). Consistent with our previous work and adopted philosophy, in the following we will call our classification "morphological". The reader should keep in mind that other authors, including the quoted work above, would, however, define our classification as a 
"structural" one. Furthermore, in our discussion below, we will distinguish between "morphology" and "structure" to indicate respectively light-weighted and mass-weighted $B / T$ values.

For the present analysis, we divide galaxies into three morphological bins, based on the basic ZENS morphological classes described in Paper II. These three bins are here referred to as the early-type, disk-dominated, and bulgeless disk galaxies. With reference to the morphological classes defined in Paper II, the early-type bin includes both elliptical galaxies, that is, galaxies described by one single component with Sérsic index $n>3$, and bulge-dominated disks with $B / T \geqslant 0.5$. The disk-dominated bin is composed of intermediate-type disks with $0.2 \leqslant B / T<0.5$, that is, of disk galaxies with a substantial but not dominant bulge component, and the bulgeless bin consists of late-type disks with $B / T<0.2$.

We note here that, at the galaxy mass scales of this study, bulgeless disks contribute negligibly to the morphological mix of the quenched galaxy population. Therefore, in the following we will simplify the presentation of our results by reporting only the fractional weight $f_{Q_{\mathrm{ETG}}}$ of the quenched early-type satellite population, relative to the whole quenched satellite population of similar mass. Given the paucity of bulgeless disks within the $M_{\text {galaxy }} \geqslant 10^{10} M_{\odot}$ quenched satellite population, a value of $\left(1-f_{Q_{\mathrm{ETG}}}\right)$ gives, in practice, the contribution of the aforementioned disk-dominated galaxies to the total quenched satellite population. Ellipticals represent instead between $5 \%$ and $15 \%$ of the whole earlytype population in the two mass bins considered in the following.

\subsection{The Final Sample for the Present Analysis}

The completeness mass limit of $10^{10} M_{\odot}$ for quenched galaxies motivates our adoption of this value as the lower galaxy mass limit in this study. We thus conduct our analyses in two well-defined bins of galaxy stellar mass: a low galaxy stellar mass bin defined by $10^{10} M_{\odot} \leqslant M_{\text {galaxy }} \leqslant 10^{10.7} M_{\odot}$ and a high-mass bin, $10^{10.7} M_{\odot} \leqslant M_{\text {galaxy }} \leqslant 10^{11.5} M_{\odot}$. Where necessary, we will refer to these mass bins with the abbreviated notation $\log M \in[10,10.7$ [ and $\log M \in[10.7,11.5]$. The median galaxy mass values of our samples in these two mass bins are respectively $10^{10.32} M_{\odot}$ and $10^{10.85} M_{\odot}$. The explored range of galaxy stellar mass straddles the characteristic (and seemingly invariant) value of $M *$ of the Schechter (1976) function that describes both the quenched and star-forming galaxy populations. As discussed in Peng et al. (2010), $M *$ emerges as the characteristic mass in the quenching of galaxies. With our convention for defining stellar masses, $M^{*} \sim 10^{10.85} M_{\odot}$, which matches the median value of our galaxy sample in the high galaxy stellar mass bin.

As shown in the Appendix, the fraction of quenched satellites and the morphological mix of these quenched satellites remain practically unchanged, in any environment, with the inclusion or exclusion of the unrelaxed groups in the computations. To maximize the size of the different galaxy samples, we will therefore use the entire ZENS group sample, independent of the group dynamical state, in studying the dependence on the different environments of the quenched satellite fraction and its morphological composition (Sections 3 and 4).

\subsection{Definition of Errors on Fractional Quantities}

Our analysis below includes estimates for the fraction $f_{Q}$ of quenched satellites and the fraction $f_{Q \text {,ETG }}$ of quenched satellites that have an early-type morphology. In order to include the impact of interloper galaxies on these fractions, we used our estimated average $\sim 20 \%-40 \%$ contamination by interlopers in the selection of group galaxy members (Paper I). Specifically, we generated 100 sample realizations for each mass and environmental bin in our study, in each of which we rejected a random $20 \%$ up to $40 \%$ of the galaxies before recalculating the quenched (and quenched early-type) fractions. We then measured the dispersion around the median fractions of all these realizations as an estimate for the impact of interlopers on these quantities. In the following, the quoted uncertainties on the measured fractions include both binomial statistics errors (Gehrels 1986) and these systematic contributions to the errors, which have been added in quadrature.

\section{QUENCHING WITHIN HALOS}

In Table 1 we list the fraction $f_{Q}$ of quenched satellites and the fraction $f_{Q, \text { ETG }}$ of quenched satellites that have an earlytype morphology, in different environmental bins of the $M_{\mathrm{Halo}}-$ $R$ parameter space. Specifically we computed these fractions in nine and six environmental bins for the low and high galaxy stellar mass bins, respectively. The nine environmental bins at low galaxy stellar mass are defined by $M_{\text {Halo }}=[12.2,13.2]$, [13.2, 13.7], and $[13.7,14.8]$ and $R \leqslant 0.5 R_{\mathrm{vir}}$, $0.5 R_{\text {vir }}<R \leqslant R_{\text {vir }}$, and $R>R_{\text {vir }}$; the six environmental bins at high stellar mass have a similar split in $R$ but a two-bin split in halo mass, i.e., $M_{\text {Halo }}=[12.8,13.7]$ and $[13.7,14.8]$ (since, as a consequence of the sample selection criteria, there are no such massive galaxies in ZENS at lower halo masses). These environmental bins are illustrated, for the low and high stellar mass bins, in the left and right panels of Figures 10 and 11, respectively.

The choice of splitting the ZENS sample on the basis of both the group-centric distance and the halo mass is not only motivated by the scientific question of which is the most fundamental parameter in determining the quenched fraction, but also by the possibility of keeping biases and correlations between the two environment descriptors under control. For example and as illustrated in Figure 10, the population of galaxies with $10^{10.7} M_{\odot}<M_{\text {galaxy }}<10^{11.5} M_{\odot}$ found at large distances from the group centers $\left(R>R_{\text {vir }}\right)$ is dominated by galaxies located in low-mass groups. This could either reflect a young dynamical state in such groups or an ambiguous identification of the central galaxy as a consequence of membership incompleteness and contamination, as extensively discussed in Paper 1. The analysis of radial effects at fixed halo mass provides a way to assess how the global trends, integrated over all halo masses, are affected by such biases.

To show the insensitivity of the quenched fractions and their morphological mix to the precise group sample selection, Table 1 also reports the fractions that we obtain by using different group samples: all relaxed groups, the "clean" sample of relaxed groups (see Section 2.1.1), and the full ZENS group sample, which also includes the unrelaxed groups. As discussed in Section 2 and the Appendix, we base the following analysis on the entire relaxed plus unrelaxed group sample. 
Table 1

Fraction $f_{Q}$ of Quenched Satellites and $f_{Q \mathrm{ETG}}$ of Quenched Satellites with an Early-type Morphology in the $M_{\mathrm{Halo}}-R / R_{\mathrm{vir}}$ Bins of Figures 10 and 11

\begin{tabular}{|c|c|c|c|c|c|c|c|}
\hline \multirow[b]{2}{*}{$\log _{10}\left(M / M_{\odot}\right) \rightarrow$} & \multicolumn{4}{|c|}{$10^{10} \leqslant \log _{10}\left(M / M_{\odot}\right)<10^{10.7}$} & \multicolumn{3}{|c|}{$10^{10.7} \leqslant \log _{10}\left(M / M_{\odot}\right) \leqslant 10^{11.5}$} \\
\hline & All $M_{\text {Halo }}$ & {$[12.2,13.2[$} & {$[13.2,13.7[$} & {$[13.7,14.8]$} & All $M_{\text {Halo }}$ & {$[12.8,13.7[$} & {$[13.7,14.8]$} \\
\hline \multicolumn{8}{|l|}{$\%$ quenched $\left[f_{Q}\right]$} \\
\hline All $R$ & $\cdots$ & $\begin{array}{c}0.58_{-0.08}^{+0.08}(0.56) \\
0.54_{-0.06}^{+0.06}\end{array}$ & $\begin{array}{c}0.56_{-0.07}^{+0.07}(0.57) \\
0.50_{-0.05}^{+0.05}\end{array}$ & $\begin{array}{c}0.54_{-0.04}^{+0.04}(0.59) \\
0.51_{-0.04}^{+0.04}\end{array}$ & $\cdots$ & $\begin{array}{c}0.69_{-0.13}^{+0.16}(0.71) \\
0.73_{-0.10}^{+0.10}\end{array}$ & $\begin{array}{c}0.77_{-0.07}^{+0.06}(0.80) \\
0.75_{-0.07}^{+0.05}\end{array}$ \\
\hline$R \leqslant 0.5 R_{200}$ & $\begin{array}{c}0.65_{-0.04}^{+0.05}(0.68) \\
0.61_{-0.04}^{+0.05}\end{array}$ & $\begin{array}{c}0.55_{-0.12}^{+0.13}(0.53) \\
0.56_{-0.13}^{+0.11}\end{array}$ & $\begin{array}{c}0.84_{-0.11}^{+0.09}(0.87) \\
0.68_{-0.10}^{+0.09}\end{array}$ & $\begin{array}{c}0.62_{-0.07}^{+0.06}(0.67) \\
0.60_{-0.06}^{+0.06}\end{array}$ & $\begin{array}{c}0.88_{-0.08}^{+0.06}(0.87) \\
0.87_{-0.07}^{+0.06}\end{array}$ & $\begin{array}{c}0.75_{-0.20}^{+0.16}(0.72) \\
0.77_{-0.24}^{+0.12}\end{array}$ & $\begin{array}{c}0.93_{-0.09}^{+0.05}(0.92) \\
0.90_{-0.08}^{+0.06}\end{array}$ \\
\hline $0.5<R \leqslant R_{200}$ & $\begin{array}{c}0.52_{-0.05}^{+0.06}(0.52) \\
0.48_{-0.05}^{+0.05}\end{array}$ & $\begin{array}{c}0.61_{-0.14}^{+0.13}(0.59) \\
0.55_{-0.13}^{+0.12}\end{array}$ & $\begin{array}{c}0.42_{-0.10}^{+0.12}(0.41) \\
0.39_{-0.09}^{+0.10}\end{array}$ & $\begin{array}{c}0.54_{-0.07}^{+0.07}(0.56) \\
0.50_{-0.06}^{+0.06}\end{array}$ & $\begin{array}{c}0.71_{-0.11}^{+0.10}(0.78) \\
0.76_{-0.08}^{+0.10}\end{array}$ & $\begin{array}{c}0.65_{-0.36}^{+0.20}(0.80) \\
0.77_{-0.20}^{+0.19}\end{array}$ & $\begin{array}{c}0.73_{-0.14}^{+0.10}(0.77) \\
0.76_{-0.10}^{+0.11}\end{array}$ \\
\hline$R>R_{200}$ & $\begin{array}{c}0.45_{-0.07}^{+0.07}(0.49) \\
0.45_{-0.05}^{+0.05}\end{array}$ & $\begin{array}{c}0.59_{-0.17}^{+0.18}(0.59) \\
0.53_{-0.09}^{+0.10}\end{array}$ & $\begin{array}{c}0.47_{-0.12}^{+0.13}(0.48) \\
0.46_{-0.08}^{+0.09}\end{array}$ & $\begin{array}{c}0.38_{-0.10}^{+0.10}(0.45) \\
0.36_{-0.08}^{+0.09}\end{array}$ & $\begin{array}{c}0.22_{-0.17}^{+0.21}(0.28) \\
0.47_{-0.15}^{+0.13}\end{array}$ & $\begin{array}{c}0.46_{-0.36}^{+0.46}(0.46) \\
0.66_{-0.17}^{+0.24}\end{array}$ & $\begin{array}{c}0.14_{-0.12}^{+0.22}(0.20) \\
0.33_{-0.16}^{+0.20}\end{array}$ \\
\hline
\end{tabular}

$\%$ quenched ETG $\left[f_{Q_{\mathrm{ETG}}}\right]$

\begin{tabular}{|c|c|c|c|c|c|c|c|}
\hline All $R$ & $\cdots$ & $\begin{array}{c}0.50_{-0.11}^{+0.12}(0.47) \\
0.54_{-0.09}^{+0.08}\end{array}$ & $\begin{array}{c}0.49_{-0.09}^{+0.10}(0.45) \\
0.54_{-0.07}^{+0.07}\end{array}$ & $\begin{array}{c}0.52_{-0.05}^{+0.06}(0.52) \\
0.51_{-0.05}^{+0.05}\end{array}$ & $\ldots$ & $\begin{array}{c}0.68_{-0.20}^{+0.15}(0.66) \\
0.78_{-0.13}^{+0.10}\end{array}$ & $\begin{array}{c}0.72_{-0.10}^{+0.08}(0.70) \\
0.69_{-0.08}^{+0.07}\end{array}$ \\
\hline$R \leqslant 0.5 R_{200}$ & $\begin{array}{c}0.51_{-0.06}^{+0.07}(0.49) \\
0.51_{-0.06}^{+0.06}\end{array}$ & $\begin{array}{c}0.47_{-0.18}^{+0.18}(0.43) \\
0.51_{-0.17}^{+0.16}\end{array}$ & $\begin{array}{c}0.49_{-0.15}^{+0.14}(0.46) \\
0.51_{-0.14}^{+0.12}\end{array}$ & $\begin{array}{c}0.53_{-0.08}^{+0.09}(0.52) \\
0.51_{-0.08}^{+0.09}\end{array}$ & $\begin{array}{c}0.71_{-0.11}^{+0.08}(0.67) \\
0.68_{-0.11}^{+0.09}\end{array}$ & $\begin{array}{c}0.61_{-0.19}^{+0.26}(0.55) \\
0.65_{-0.20}^{+0.25}\end{array}$ & $\begin{array}{c}0.74_{-0.09}^{+0.11}(0.71) \\
0.69_{-0.11}^{+0.10}\end{array}$ \\
\hline $0.5<R \leqslant R_{200}$ & $\begin{array}{c}0.52_{-0.08}^{+0.07}(0.50) \\
0.55_{-0.07}^{+0.07}\end{array}$ & $\begin{array}{c}0.47_{-0.19}^{+0.18}(0.43) \\
0.51_{-0.19}^{+0.16}\end{array}$ & $\begin{array}{c}0.39_{-0.17}^{+0.20}(0.33) \\
0.47_{-0.15}^{+0.17}\end{array}$ & $\begin{array}{c}0.57_{-0.11}^{+0.09}(0.63) \\
0.59_{-0.10}^{+0.09}\end{array}$ & $\begin{array}{c}0.69_{-0.14}^{+0.12}(0.70) \\
0.68_{-0.11}^{+0.11}\end{array}$ & $\begin{array}{c}0.76_{-0.41}^{+0.24}(0.76) \\
0.73_{-0.25}^{+0.21}\end{array}$ & $\begin{array}{c}0.67_{-0.13}^{+0.16}(0.68) \\
0.67_{-0.14}^{+0.12}\end{array}$ \\
\hline$R>R_{200}$ & $\begin{array}{c}0.48_{-0.12}^{+0.11}(0.48) \\
0.52_{-0.08}^{+0.07}\end{array}$ & $\begin{array}{c}0.61_{-0.29}^{+0.19}(0.61) \\
0.59_{-0.14}^{+0.14}\end{array}$ & $\begin{array}{c}0.59_{-0.21}^{+0.17}(0.55) \\
0.61_{-0.12}^{+0.12}\end{array}$ & $\begin{array}{c}0.31_{-0.13}^{+0.20}(0.33) \\
0.30_{-0.13}^{+0.16}\end{array}$ & $\begin{array}{c}1.00_{-0.66}(1.00) \\
0.90_{-0.17}^{+0.10}\end{array}$ & $\begin{array}{c}1.00_{-0.84}(1.00) \\
1.00_{-0.26}\end{array}$ & $\begin{array}{c}1.00_{-0.84}(1.00) \\
0.75_{-0.31}^{+0.25}\end{array}$ \\
\hline
\end{tabular}

Note. The table shows the fraction $f_{Q}$ of quenched satellites of any morphology (top) and the fraction $f_{Q \mathrm{ETG}}$ of quenched galaxies that have an early-type morphology (bottom), in each of the quadrants in which Figures 10 and 11 split the $M_{\mathrm{Halo}}-R / R_{\mathrm{vir}}$ plane, for the two galaxy stellar mass bins of our analysis. Roman entries show values obtained considering the entire ZENS sample of relaxed plus unrelaxed groups; gray entries refer to fractions derived when using only the sample of relaxed groups. Always in italic, in parentheses, are reported the corresponding fractions for the "clean" sample of relaxed groups only: this excludes groups for which a selfconsistent solution for the central galaxy in the mass, halo-centric distance, and velocity parameter space could be found within the errors on the galaxy mass estimates, but the galaxy that satisfies these conditions is not the galaxy with the nominally highest "best-fit" stellar mass (see Section 2 and Paper I). All fractions are corrected for the effects of the $2 \mathrm{dFGRS}$ spectroscopic incompleteness; we highlight that the applied corrections are quite small. The errors indicate Bayesian $\pm 1 \sigma$ confidence intervals for a binomial distribution.

\subsection{The Dependence of the Quenched Satellite Fraction on Halo Mass and Halo-centric Distance}

Figures 1(a) and (b) show the results of Table 1 for $f_{Q}$ in a graphic form. Specifically, the upper panels of Figure 1(a) show the quenched fraction $f_{Q}$ of our lower-mass $\log M \in$ $[10,10.7$ [ satellite galaxies as a function of group halo mass $M_{\text {Halo }}$ (upper right) and normalized halo-centric radius $R / R_{\text {vir }}$ (upper left). The lower panels show the same results, this time split into the three bins of halo-centric radius (lower left plot) and the three bins of halo mass (lower right plot) that are listed above and in Table 1 (and illustrated by the dotted lines in Figure 10). Figure 1(b) shows the same for the higher mass satellite galaxies $(\log M \in[10.7,11.5])$, but now, as discussed above and in the Appendix, we split the sample in only two bins of halo mass in the lower left panel. In both figures, we plot the quenched fractions in the corresponding environmental bins, that is, the values reported in Table 1 , at the median values of the environmental parameters within the bins in question. The black dashed and dotted lines in all panels of both figures, and the red horizontal lines and hatched areas in their top-right panels, are discussed in Sections 5.1 and 5.4, respectively, where we compare the observed trends for $f_{Q}$ with classical SAMs and with the Peng et al. (2010) predictions for mass and environment quenching (see caption of Figure 1(a)).

The significance of the differences in $f_{Q}$ and $f_{Q_{\mathrm{ETG}}}$ measured across the $M_{\text {Halo }}-R$ parameter space is given in Table 3 . Specifically, we provide the $p$ value (probability of obtaining the observed difference from the same parent sample) for a $z$ test performed on the fractions measured in the lowest and highest environmental bins, for any given environmental indicator and galaxy sample considered in Figures 1(a)-2(b).

At the galaxy mass scales that we are probing, we find no significant dependence on halo mass of the quenched satellite fraction $f_{Q}$ when this is integrated over all halo-centric distances: about $50 \%$ and $70 \%$ of $\log M \in[10,10.7$ [ and $\log M \in[10.7,11.5] \quad$ satellite galaxies, respectively, are quenched systems at all halo masses. A quantitative $z$ test indicates consistency between the quenched fraction in small and massive groups for both low- and high-mass galaxies ( $p$ value greater than $60 \%$ ); bootstrapping simulations give a $1 \sigma$ upper-limit slope $\frac{d f_{Q}}{d \log M} \leqslant 0.1$ for the $f_{Q}$ versus $M_{\text {Halo }}$ relation across the range covered by our data. In Paper I, we estimated a typical uncertainty of about 0.3 dex for the halo masses, which in general would tend to weaken any actual dependence of parameters on halo mass, even though the width of the halo mass bins in our analysis is larger than this typical uncertainty. Mock simulations, discussed in Paper I, indicate that the observed flat relation between $f_{Q}$ and $M_{\text {Halo }}$ does imply a negligible intrinsic slope for this relationship. We note that, in their SDSS analysis, Peng et al. (2012) find that the fraction of quenched satellites is invariant with halo mass at fixed local overdensity, while Woo et al. (2013) find a weak positive correlation between their satellite red fraction and halo mass. 
$10^{10} \leq \mathrm{M}_{\text {galaxy }} / \mathrm{M}_{\odot}<10^{10.7}$

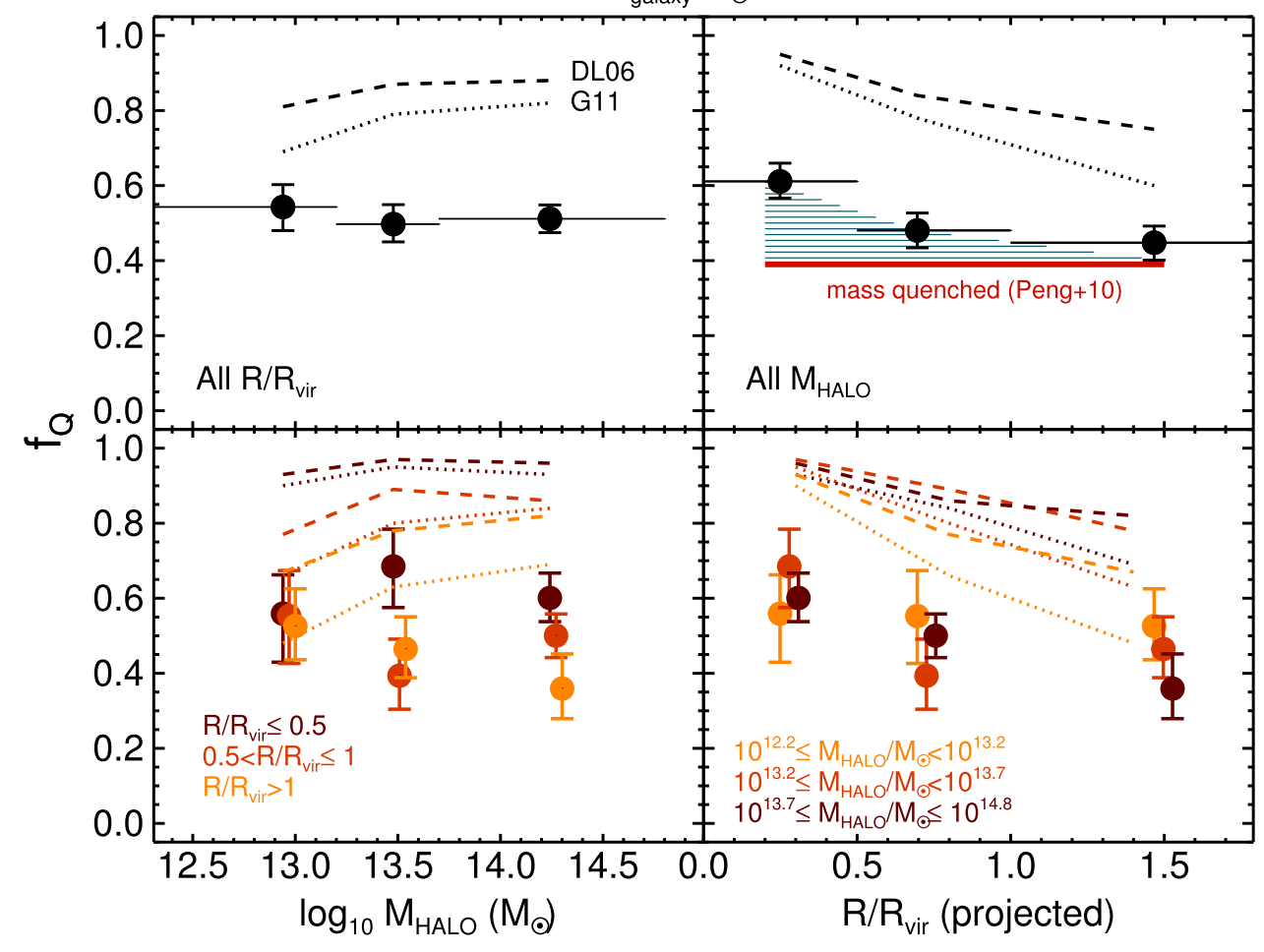

$\mathrm{b}$

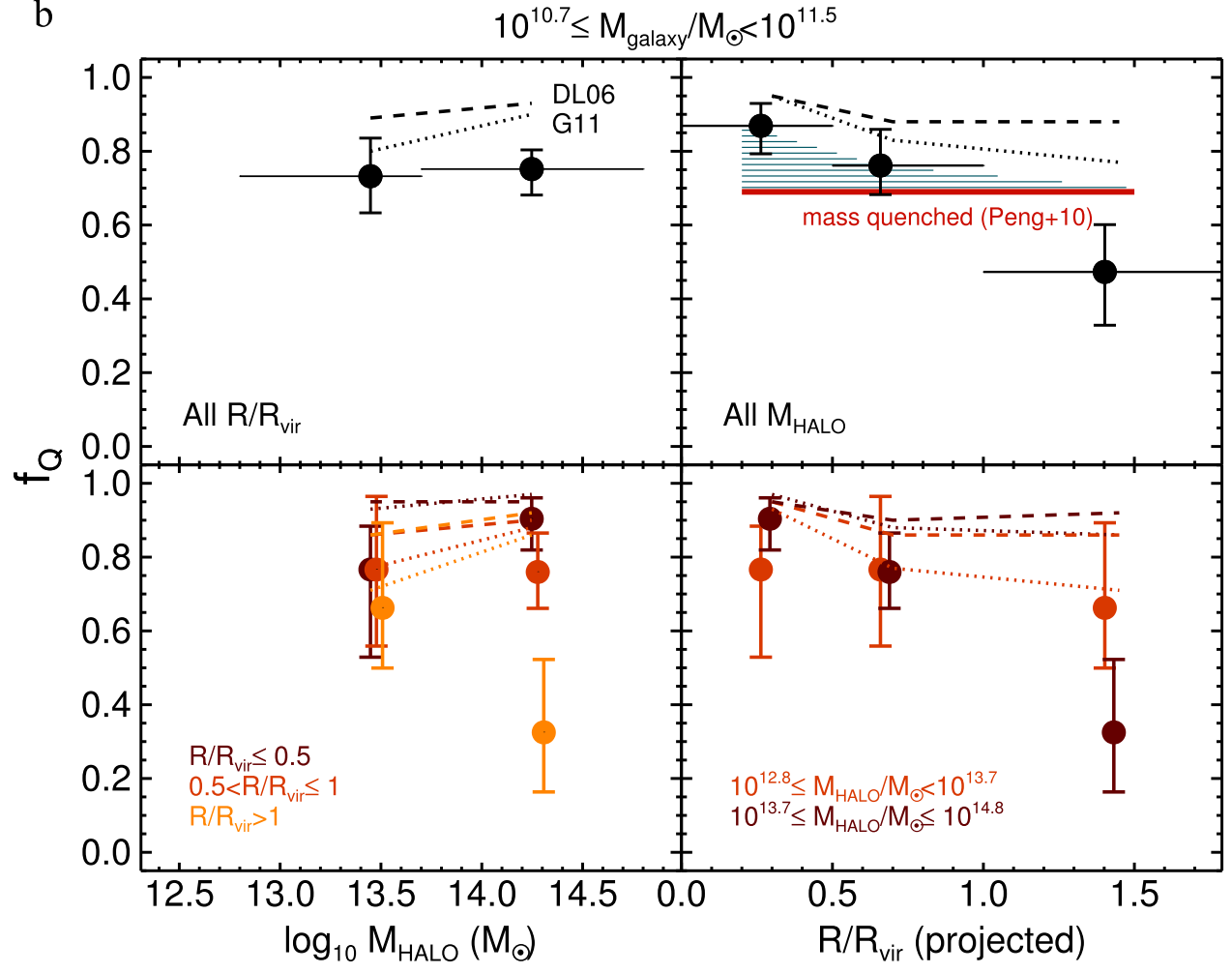

Figure 1. Top panels: fraction $f_{Q}$ of quenched satellite galaxies of all morphologies with stellar mass in the range $\log M \in[10,10.7$ [, as a function of halo mass (left) and halo-centric distance (right). Both relaxed and unrelaxed groups are included in the sample (see discussion in the Appendix). The vertical error bars indicate Bayesian $\pm 1 \sigma$ confidence intervals for a binomial distribution. In the upper panels, satellites at all halo-centric distances (left) or at all halo masses (right) are included in the sample, and the horizontal bars show the width of the given environmental bin (the same bins are also considered in the lower panels and hence not shown again). The red horizontal line in the top-right panel indicates the level of mass quenching as expected from the Peng et al. (2010) model, and the hatched area above the red horizontal line highlights the quenching excess that we attribute to environmental quenching (see discussion in Section 5.1). The bottom panels show the quenched fractions of satellite galaxies, this time split over the bins in the second environmental parameter (i.e., bins of $R / R_{\mathrm{vir}}$ for the plot of $f_{O}$ vs. $M_{\mathrm{Halo}}$, and bins of $M_{\text {Halo }}$ for the plot of $f_{Q}$ vs. $R / R_{\text {vir }}$ ). Colors range from light (yellow) to dark (brown) moving from sparse (low $M_{\text {Halo }}$, high $R / R_{\text {vir }}$ ) to dense (high $M_{\text {Halo }}$, low $R / R_{\text {vir }}$ ) environments (see legend). The black lines show the predictions of the semianalytic models of De Lucia et al. 2006 (DL06, dashed line) and Guo et al. 2011 (G11, dotted line), which are discussed in Section 5.4. In the bottom panels, the models are split in bins of the second environmental parameter, as done for the ZENS data points. Bottom panels: as in top panels, but for the galaxy stellar mass bin $10^{10.7}-10^{11.5} M_{\odot}$. 

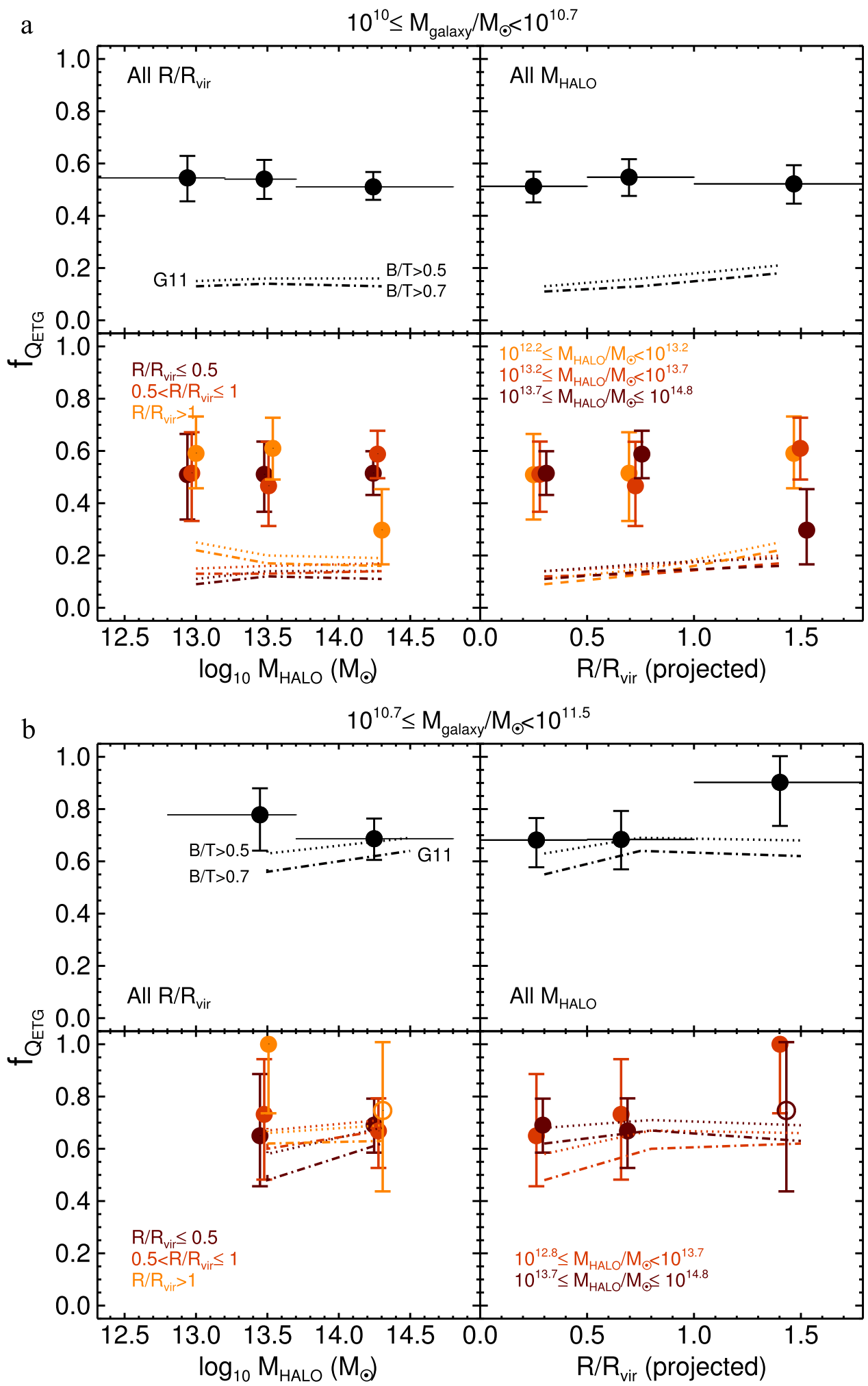

Figure 2. Top panels: fraction of quenched satellite galaxies with an early-type morphology as a function of group halo mass $M_{\mathrm{Halo}}$ (left panel) and halo-centric distance $R / R_{\text {vir }}$ (right panel), in the galaxy stellar mass bin $10^{10}-10^{10.7} M_{\odot}$. Both relaxed and unrelaxed groups are included in the sample (see discussion in the Appendix). In the upper panels, satellites at all halo-centric distances (left) and at all halo masses (right) are included in the sample. The bottom panels show again the quenched fractions of satellite galaxies with an early-type morphology relative to $f_{Q}$, this time split over the bins in the second environmental parameter, which are defined by the dotted lines of Figure 11 (bins of $R / R_{\mathrm{vir}}$ for the plot of $f_{Q_{\mathrm{ETG}}}$ vs. $M_{\mathrm{Halo}}$, and bins of $M_{\mathrm{Halo}}$ for the plot of $f_{O_{\mathrm{ETG}}}$ vs. $R / R_{\mathrm{vir}}$ ). Colors range from light (yellow) to dark (brown) moving from sparse (low $M_{\text {Halo }}$, high $R / R_{\mathrm{vir}}$ ) to dense (high $M_{\text {Halo }}$, low $R / R_{\mathrm{vir}}$ ) environments. The black lines show the predictions of the semianalytic models of Guo et al. 2011 (G11) that we discuss in Section 5.4. Dash-dotted lines indicate model early-type satellite galaxies defined as systems with a $B / T>0.7$ in stellar mass; the dotted lines are for model satellites with stellar mass of $B / T>0.5$. The models in the bottom panels are split in bins of the second environmental parameter, as done for the ZENS data. Bottom panels: as in top panels, but for the galaxy stellar mass bin $10^{10.7}-10^{11.5} M_{\odot}$. In the lower panels, open symbols indicate $\leqslant 5$ objects at the denominator of the given fraction. 
Consistent with other independent analyses, our sample shows instead a significant environmental dependence of $f_{Q}$ on halo-centric distance $R$ : the quenched fraction, averaged over all halo masses, decreases with increasing $R$ (see upper right plots of Figures 1(a) and (b)). This is also seen in the SDSS analysis of Woo et al. (2013), and, indirectly, of Peng et al. (2012) if their local projected density $\rho$ is interpreted as a proxy for the halo-centric radius; other studies have also found a similar trend (e.g., Rasmussen et al. 2012; George et al. 2013). In our data, this radial trend is seen mostly, and most strongly, at the highest halo masses. When averaged over all halo mass scales, the fraction $f_{Q}$ of low-mass quenched satellites in our sample declines from $\gtrsim 60 \%$ in the cores of groups down to $\lesssim 50 \%$ at the virial radius, whereas for the high-mass satellites it decreases from $\gtrsim 80 \%$ down to $\lesssim 60 \%$. The total quenched fraction keeps decreasing at radii larger than $R_{\mathrm{vir}}$ in the high galaxy mass bin where our $R>R_{\text {vir }}$ measurement gives $f_{Q} \sim 50 \%$. In the low-mass bin, the quenched satellite fraction flattens outside the virial radius; for both galaxy mass bins, however, a two-sided $z$ test shows a $<1 \%$ probability that the inner $\left(R / R_{\text {vir }} \leqslant 0.5\right)$ and outer $\left(R / R_{\text {vir }}>1\right)$ measured quenched fractions could be consistent with one another.

In the ZENS sample, the results that are obtained when considering only galaxies located at large halo-centric distances $R>R_{\text {vir }}$ or in low-mass halos differ from the global trends that we have discussed so far when integrating over all $R_{\mathrm{vir}}$ or $M_{\mathrm{Halo}}$ (see bottom panels of Figures 1(a) and (b)). Low-mass groups, in fact, do not display a clear radial variation of $f_{Q}$ in both galaxy mass bins here studied. The flattening of $f_{Q}$ versus $R / R_{\text {vir }}$ for $10^{10} M_{\odot}<M_{\text {galaxy }}<10^{10.7} M_{\odot}$ galaxies is driven by groups with $M_{\text {Halo }}<10^{13.2} M_{\odot}$ (a stronger radial dependence is observed in more massive groups). The lack of strong radial variations of $f_{Q}$ at low halo masses may be genuinely due to the inability of these environments (e.g., due to a low intragroup medium density) to produce environmental effects. The possibility remains, however, that this may simply reflect the difficulty of identifying galaxy members, including the central galaxy, in these small, loose groups (see also above and the discussion in Paper I regarding the uncertainties affecting the definition of the group centers). Mixing of the inner and outer satellite populations could also be causing the mild decrease in $f_{Q}$ with increasing group mass for galaxies located at large halo-centric distances in low-mass groups (see lower left panels of Figures 1(a) and (b)).

\subsection{The Dependence of the Morphological Mix of the Quenched Satellite Fraction on Halo Mass and Halo-centric Distance}

Figures 2(a) and (b) show in graphic form the results of Table 1 for the fraction of quenched satellites that have earlytype morphology, $f_{Q_{\mathrm{ETG}}}$. Specifically, they show the morphological composition of the quenched satellite population for the two bins of satellite stellar mass, as functions of halo mass and halo-centric radius. The black (dashed, dot-dashed, and dotted) lines in both figures are again the predictions of classical SAMS, which we discuss in Section 5.4.

As commented in Section 2.4, in ZENS there are virtually no bulgeless quenched galaxies at the galaxy mass scale that we are studying, and the quenched satellites consist therefore largely of intermediate-type disks with a dominant disk component but a nonnegligible bulge and of a few elliptical galaxies.
Within the errors, we find no trends in the morphological mix of the quenched satellite population with either halo mass or halo-centric radius, despite the significant radial trends seen in the total fraction of quenched satellites in Figures 1(a) and (b). All of the data points in Figures 2(a) and (b) are consistent with a constant early-type fraction of about $50 \%$ for $\log M \in$ $[10,10.7[$ satellite galaxies, and about $65 \%$ for the more massive $\log M \in[10.7,11.5]$ ones. Thus, while it is clear from Figures 1(a) and (b) that more satellites have been quenched in the cores of groups than in their outskirts, the relative fractions of early-type and disk-dominated morphologies in the quenched satellite population do not change with group-centric distance. Similarly, no significant variation is observed in the fraction of elliptical galaxies among all quenched galaxies: we measure constant values of about 5\%-10\% and $20 \%$ in the two galaxy mass bins across most environments. The only exception is for $\log M \in[10.7,11.5]$ galaxies at $R>R_{\mathrm{vir}}$, in which ellipticals represent $50 \%$ of the quenched population and cause the slight upturn in $f_{Q_{\mathrm{ETG}}}$ observed in the upper right panel of Figure 2(b). This difference is, however, only marginally significant ( $p$ value $=0.08)$, and it could be affected by contamination in the group outskirts.

The constancy of the $f_{Q_{\mathrm{ETG}}}$ with halo-centric radius in Figures 2(a) and (b) does not of course violate the well-known morphology-density relation of Dressler (1980). Our results indicate that this is due to the changing fraction of quenched galaxies with radius $f_{Q}(R)$, rather than a change in the morphologies of the quenched galaxies themselves-although, as we discuss in Sections 5.2 and 5.3, there is a systematic difference in the morphologies (but, as we argue, not in the structure) of quenched satellites and their star-forming counterparts of similar mass.

\section{INFLUENCES ON QUENCHING FROM OUTSIDE HALOS?}

We can ask whether quenching processes and possible morphological transformations may be triggered by the average LSS density on which the group halos reside and thus search for trends of $f_{Q}$ and $f_{Q_{\mathrm{ETG}}}$ with the average LSS density parameter $\delta_{\text {LSS }}$ discussed in Section 2.1.2. We recall that this parameter represents the average density at the location of a given group and is identical for all galaxies that are members of that group. Also, in order to minimize as much as possible the physical cross talk between high LSS densities and high halo masses, we exclude for this analysis the most massive halos in our sample. Specifically, as in Paper I and as noted above, we use for this analysis only groups with $M_{\text {Halo }} \leqslant 10^{13.7} M_{\odot}$. Furthermore, to keep any residual dependence on the group mass under control even within the reduced sample, we show with gray symbols in the upper panels of Figures 3(a) and 4(a) the results separately for groups above and below $M_{\text {Halo }}=10^{13.2} M_{\odot}$. As before, we use here both relaxed and unrelaxed groups satisfying this mass condition.

Taking into account the range of $\delta_{\text {LSS }}$ covered by the galaxy samples, we split the LSS density domain into three bins with boundaries at $\log _{10}\left(1+\delta_{\mathrm{LSS}}\right)=0.3$ and 0.7 for the low-mass sample and into two bins split at $\log _{10}\left(1+\delta_{\text {LSS }}\right)=0.7$ for the high-mass sample. We then compute the fractions of quenched satellites within these boundaries of LSS density in the same three bins of halo-centric distance already defined in Section 3: $R \leqslant 0.5 R_{\text {vir }}, 0.5 R_{\text {vir }}<R \leqslant R_{\text {vir }}$, and $R>R_{\text {vir. }}$. The nine (six) environmental bin splittings of the $\delta_{\text {LSS }}-R$ plane at low (high) 
a $\quad \log \left(M_{\text {HALO }} / M_{\odot}\right)<13.7 \quad 10^{10} \leq M_{\text {galaxy }} / M_{\odot}<10^{10.7}$
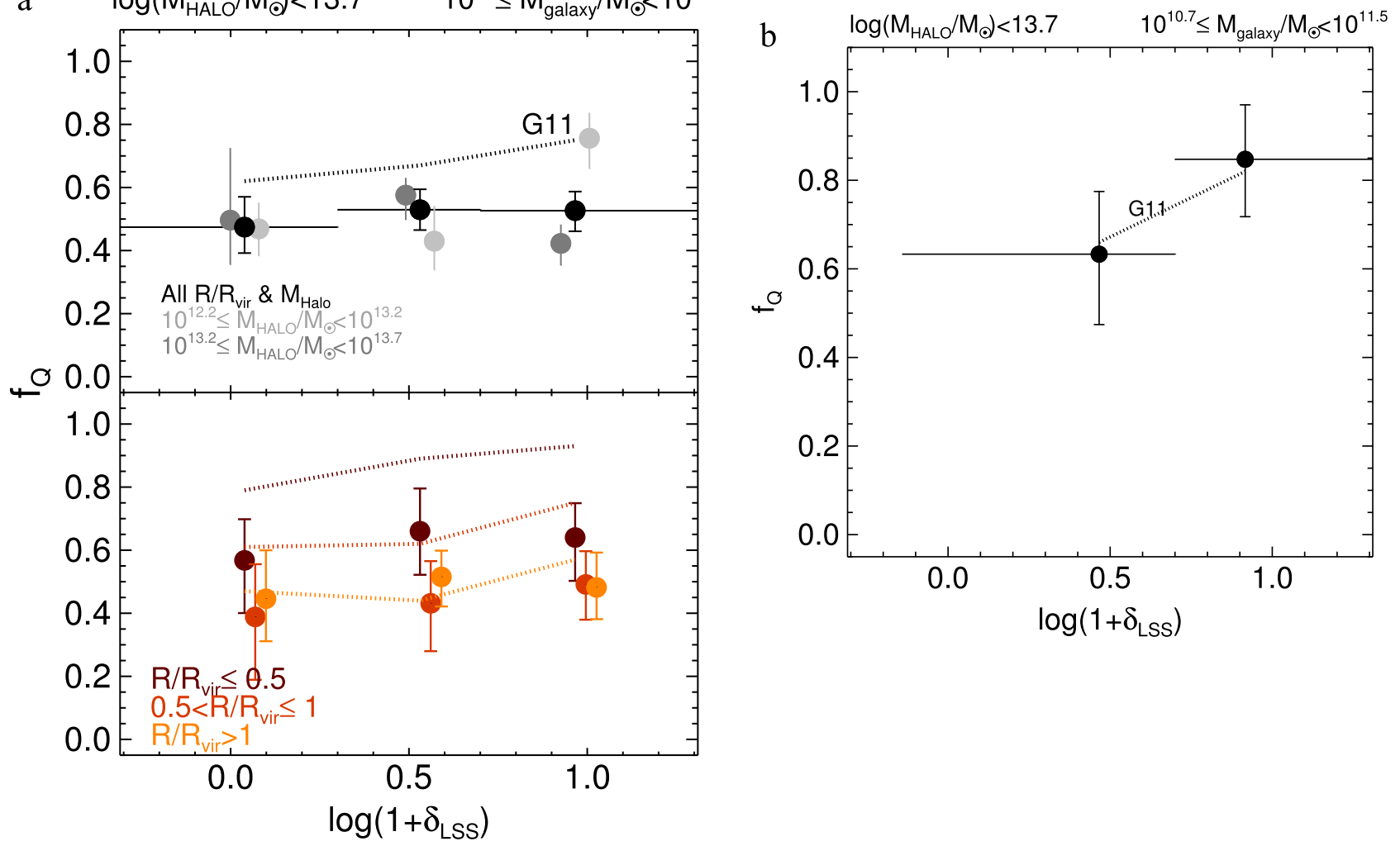

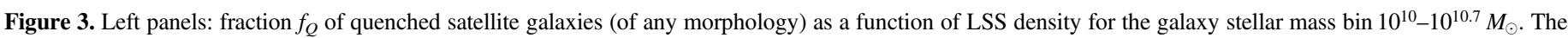

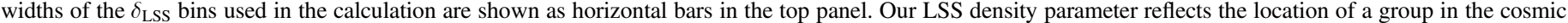

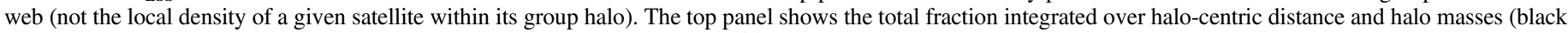

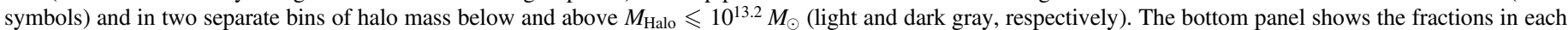

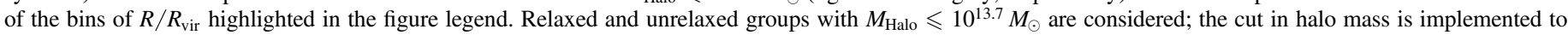

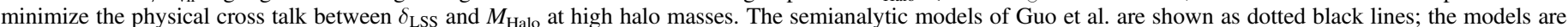

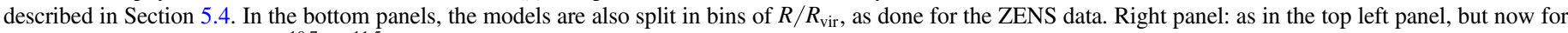
the galaxy stellar mass bin $10^{10.7}-10^{11.5} M_{\odot}$.

galaxy stellar mass are illustrated in the left (right) panel of Figure 12 of the Appendix; the corresponding quenched and quenched-ETG fractions are listed in Table 2. As done when investigating the radial and halo mass dependence, we provide in Table 3 the $z$ test $p$ values for the fractions measured in the low- and high-density bins.

We show in Figures 3(a) to 4(b) the analogous plots to the left panels of Figures 1(a) to 2(b), this time, however, using the underlying density of the cosmic LSS as the environmental parameter on the $x$ axis. At high galaxy masses, our $M_{\text {Halo }} \leqslant 10^{13.7} M_{\odot}$ ZENS sample is too small to enable for us a split in different bins of halo-centric distance (see the right panels of Figures 12 and 13) or different bins of halo mass. Thus Figures 3(b) and 4(b) are analogous plots only to the upper panels of Figures 3(a) and 4(a) with no splitting according to halo mass.

For galaxies with $10^{10.7}<M<10^{11.5} M_{\odot}$, we formally observe an increase of $f_{Q}$ with increasing LSS density, which matches the trend predicted by, for example, the Guo et al. (2011) semianalytical model (which we discuss further in Section 5.4; see the dotted line in Figure 3(b)). In contrast, the decrease of $f_{Q_{\mathrm{ETG}}}$ with increasing $\delta_{\mathrm{LSS}}$ for the most massive galaxies is opposite to that predicted by this same model (dotted and dash-dotted lines in Figure 4(b)). Given, however, that at these high masses, even in the averaged (over $R$ ) relationships of Figures 3(b) and 4(b), the statistical uncertainties are substantial, and thus the significance of the trends observed with $\delta_{\mathrm{LSS}}$ is low (we estimate an at least $20 \%$ probability that the populations in the two environmental bins have consistent fractions by applying again a $z$ test), we will not include them in the discussion of our main results in Section 5.

In the lower galaxy mass bin, we have, in contrast, enough statistics to properly explore the dependence of the quenched fraction and its morphological mix on the LSS density. When considering all groups regardless of their halo mass, we find no evidence of any impact of the LSS density at which the groups reside on the fraction of $\log M \in[10,10.7]$ quenched satellites that inhabits these groups (at any halo-centric distance; see bottom panel of Figure 3(a)). Conversely, a statistically significant increase in the quenched fraction between the lowest and highest density bins is measured when considering the low-mass groups only. The morphological mix of the quenched galaxies with $10^{10}<M<10^{10.7} M_{\odot}$ is instead independent of LSS density surrounding the halos in which these satellites reside. This is true regardless of the halo-centric distance of the satellite within the halo (see gray points and bottom panel in Figure 4(a)) or the halo mass. These results support our previous conclusions that, in spite of an increase in the overall quenched fraction in dense environments, there is no clear evidence for a variation in the typical morphology of quenched galaxies. 
a $\quad \log \left(M_{\text {HALO }} / M_{\odot}\right)<13.7 \quad 10^{10.7} \leq M_{\text {galaxy }} / M_{\odot}<10^{11.5}$
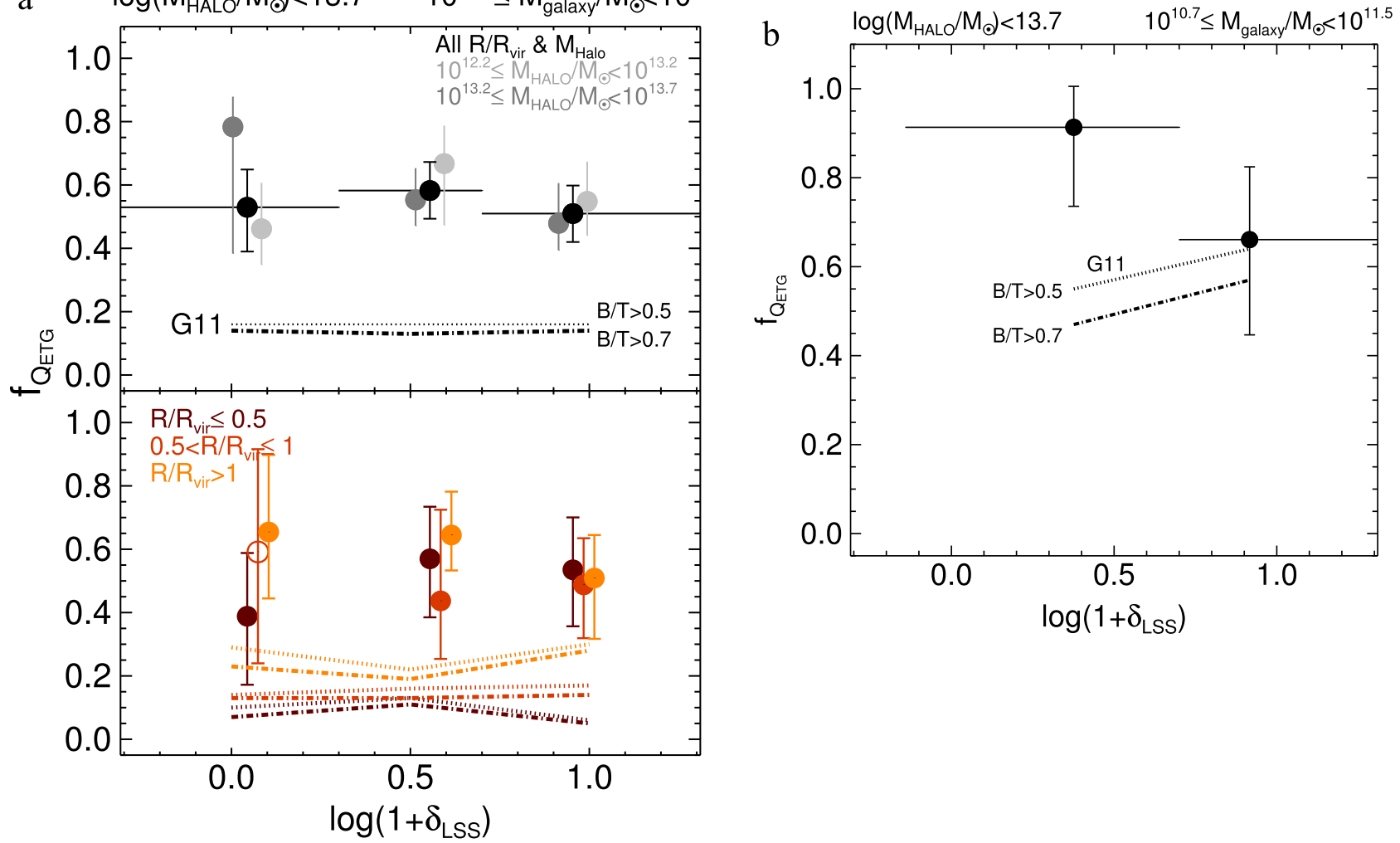

Figure 4. Left panel: fraction of quenched satellite galaxies with an early-type morphology as a function of LSS density, for the galaxy stellar mass bin $10^{10}-10^{10.7} M_{\odot}$. Our LSS density parameter reflects the location of a group in the cosmic web (not the local density of a given satellite within its group halo). The top panel shows the total fraction integrated over halo-centric distance; the bottom panel shows the fractions in each of the bins of $R / R_{\text {vir }}$ highlighted in Figure 13 . Relaxed and unrelaxed groups with $M_{\mathrm{Halo}} \leqslant 10^{13.7} M_{\odot}$ are considered. The semianalytic models of Guo et al. are shown as black lines; the models are described in Section 5.4. Different line types indicate model early-type satellites defined as systems with a $B / T>0.7$ in stellar mass (dash-dotted line); the dotted lines are for model satellite galaxies with a stellar mass $B / T>0.5$. In the bottom panels, the models are split in bins of $R / R_{\mathrm{vir}}$, as done for the ZENS data. Right panel: as in top left panel, this time for the galaxy stellar mass bin $10^{10.7}-10^{11.5} M_{\odot}$.

\section{INSIGHTS ON QUENCHING FROM MORPHOLOGIES}

The main results from the previous sections can be summarized in terms of two simple statements: (1) the quenched fraction of satellites has an inverse dependence on halo-centric distance, but does not depend on group halo mass and, especially for $M_{\text {galaxy }} \gtrsim 10^{10.7} M_{\odot}$, also on the surrounding LSS density; and (2) the morphological mix of the quenched satellites $f_{Q_{\mathrm{ETG}}}$ does not appear to depend at all on radius, halo mass, or LSS density, and therefore, by extension, is also independent of the quenched fraction $f_{Q}$. We discuss these findings below in the context of the mass and environment quenching picture of Peng et al. (2010) and in comparison with some representative traditional SAMs.

\subsection{A Similar Impact of Mass and Satellite Quenching on Morphologies}

Mass quenching is the process that controls the overall mass functions of star-forming and passive galaxies (Peng et al. 2010); it is clearly associated with the mass of the galaxy, although it is hard to distinguish whether it is the stellar mass, halo mass, or even black hole mass that is relevant (see discussion and references in Section 1). Environment quenching appears to act only on satellites (e.g., van den Bosch et al. 2008; Peng et al. 2012; Wetzel et al. 2012; Kovač et al. 2014), and such satellite quenching has been shown to be independent of stellar mass (when expressed as a differential quenching efficiency, as in Peng et al. 2010).

Given the well-known correlation of early-type morphology with mass, a simple hypothesis would have been to suppose that mass quenching produces early-type morphologies, while satellite quenching is not associated with any morphological transformation and adds quenched disk systems to the total population of passive galaxies. Based on our results, we can rule out this simple idea.

At a given stellar mass, some (constant) fraction of satellites should have been quenched by mass quenching, independent of environment (Peng et al. 2010). We would expect this to also be the $f_{Q}$ of satellites at large radii, $R>R_{\mathrm{vir}}$. This is tantamount to saying that there is no satellite quenching per se at these large radii. This is not inconsistent with reports of alleged environmental-quenching effects on galaxies at very large distances from the centers of massive clusters (e.g., an enhanced red fraction and reduced $\mathrm{H}_{\mathrm{I}}$ emission; Wetzel et al. 2014 and references therein) since these effects are largely contributed by central galaxies of lower-mass halos and disappear when satellites only are considered (Wetzel et al. 2012). Note also that, as shown in the Appendix, most of the massive satellites that are found at very large halo-centric distances are members of unrelaxed groups without a welldefined center, which casts some doubts on their identification as $R>R_{\text {vir }}$ satellites of the given halo. 
Table 2

Fraction $f_{Q}$ of Quenched Satellites and $f_{Q \text { ETG }}$ of Quenched Satellites with an Early-type Morphology as a Function of $\delta_{\text {LSS }}$ (in the Low Galaxy Mass Bin Also Split in the $R / R_{\text {vir }}$ Bins of Figures 12 and 13 )

\begin{tabular}{|c|c|c|c|c|c|}
\hline \multirow[b]{2}{*}{$\log _{10}\left(1+\delta_{\mathrm{LSS}}\right) \rightarrow$} & \multicolumn{3}{|c|}{$10^{10} \leqslant \log _{10}\left(M / M_{\odot}\right)<10^{10.7}$} & \multicolumn{2}{|c|}{$10^{10.7} \leqslant \log _{10}\left(M / M_{\odot}\right) \leqslant 10^{11.5}$} \\
\hline & {$[-0.5,0.3[$} & {$[0.3,0.7[$} & {$[0.7,2]$} & {$[-0.5,0.7[$} & {$[0.7,2]$} \\
\hline \multicolumn{6}{|l|}{$\%$ Quenched $\left[f_{Q}\right]$} \\
\hline All $R$ & $\begin{array}{c}0.48_{-0.10}^{+0.11}(0.48) \\
0.47_{-0.08}^{+0.09}\end{array}$ & $\begin{array}{c}0.63_{-0.10}^{+0.09}(0.61) \\
0.53_{-0.07}^{+0.07}\end{array}$ & $\begin{array}{c}0.57_{-0.07}^{+0.07}(0.59) \\
0.53_{-0.07}^{+0.06}\end{array}$ & $\begin{array}{c}0.60_{-0.27}^{+0.16}(0.60) \\
0.63_{-0.16}^{+0.14}\end{array}$ & $\begin{array}{c}0.77_{-0.26}^{+0.15}(0.85) \\
0.85_{-0.13}^{+0.13}\end{array}$ \\
\hline$R \leqslant 0.5 R_{200}$ & $\begin{array}{c}0.55_{-0.19}^{+0.16}(0.55) \\
0.57_{-0.17}^{+0.13}\end{array}$ & $\begin{array}{c}0.77_{-0.19}^{+0.11}(0.76) \\
0.66_{-0.13}^{+0.13}\end{array}$ & $\begin{array}{c}0.75_{-0.16}^{+0.10}(0.78) \\
0.64_{-0.14}^{+0.11}\end{array}$ & $\begin{array}{l}\cdots \\
\cdots\end{array}$ & $\begin{array}{l}\cdots \\
\ldots\end{array}$ \\
\hline $0.5<R \leqslant R_{200}$ & $\begin{array}{c}0.36_{-0.21}^{+0.19}(0.36) \\
0.39_{-0.20}^{+0.17}\end{array}$ & $\begin{array}{c}0.55_{-0.20}^{+0.17}(0.51) \\
0.43_{-0.14}^{+0.13}\end{array}$ & $\begin{array}{c}0.52_{-0.11}^{+0.13}(0.52) \\
0.49_{-0.11}^{+0.11}\end{array}$ & $\begin{array}{l}\cdots \\
\cdots\end{array}$ & $\begin{array}{l}\cdots \\
\cdots\end{array}$ \\
\hline$R>R_{200}$ & $\begin{array}{c}0.52_{-0.21}^{+0.26}(0.52) \\
0.45_{-0.14}^{+0.16}\end{array}$ & $\begin{array}{c}0.56_{-0.18}^{+0.17}(0.56) \\
0.52_{-0.10}^{+0.09}\end{array}$ & $\begin{array}{c}0.46_{-0.13}^{+0.17}(0.48) \\
0.48_{-0.10}^{+0.11}\end{array}$ & $\begin{array}{l}\cdots \\
\cdots\end{array}$ & $\begin{array}{l}\cdots \\
\cdots\end{array}$ \\
\hline \multicolumn{6}{|c|}{$\%$ Quenched ETG $\left[f_{Q_{\mathrm{ETG}}}\right]$} \\
\hline All $R$ & $\begin{array}{c}0.48_{-0.15}^{+0.19}(0.48) \\
0.53_{-0.14}^{+0.12}\end{array}$ & $\begin{array}{c}0.50_{-0.11}^{+0.14}(0.46) \\
0.58_{-0.09}^{+0.09}\end{array}$ & $\begin{array}{c}0.49_{-0.11}^{+0.10}(0.45) \\
0.51_{-0.09}^{+0.09}\end{array}$ & $\begin{array}{c}0.84_{-0.32}^{+0.16}(0.84) \\
0.91_{-0.18}^{+0.09}\end{array}$ & $\begin{array}{c}0.56_{-0.21}^{+0.29}(0.49) \\
0.66_{-0.22}^{+0.17}\end{array}$ \\
\hline$R \leqslant 0.5 R_{200}$ & $\begin{array}{c}0.36_{-0.23}^{+0.22}(0.36) \\
0.39_{-0.21}^{+0.20}\end{array}$ & $\begin{array}{c}0.54_{-0.17}^{+0.22}(0.50) \\
0.57_{-0.18}^{+0.16}\end{array}$ & $\begin{array}{c}0.50_{-0.18}^{+0.18}(0.47) \\
0.54_{-0.18}^{+0.17}\end{array}$ & $\begin{array}{l}\cdots \\
\ldots\end{array}$ & $\begin{array}{l}\cdots \\
\cdots\end{array}$ \\
\hline $0.5<R \leqslant R_{200}$ & $\begin{array}{c}0.49_{-0.34}^{+0.37}(0.49) \\
0.59_{-0.35}^{+0.32}\end{array}$ & $\begin{array}{c}0.43_{-0.28}^{+0.23}(0.33) \\
0.44_{-0.19}^{+0.29}\end{array}$ & $\begin{array}{c}0.42_{-0.17}^{+0.17}(0.37) \\
0.49_{-0.17}^{+0.15}\end{array}$ & $\begin{array}{l}\cdots \\
\cdots\end{array}$ & $\begin{array}{l}\cdots \\
\cdots\end{array}$ \\
\hline$R>R_{200}$ & $\begin{array}{c}0.70_{-0.44}^{+0.22}(0.70) \\
0.65_{-0.21}^{+0.24}\end{array}$ & $\begin{array}{c}0.51_{-0.28}^{+0.18}(0.51) \\
0.64_{-0.11}^{+0.14}\end{array}$ & $\begin{array}{c}0.62_{-0.25}^{+0.19}(0.57) \\
0.51_{-0.19}^{+0.13}\end{array}$ & $\begin{array}{l}\cdots \\
\cdots\end{array}$ & $\begin{array}{l}\cdots \\
\cdots\end{array}$ \\
\hline
\end{tabular}

Note. Note that in this analysis only groups with $M_{\text {Halo }} \leqslant 10^{13.7} M_{\odot}$ are considered. All entries are coded as in Table 1 .

Using the quenching efficiencies for mass quenching of Peng et al. (2010) and taking into account a $\sim 0.2$ dex offset between their stellar masses (actual, including return to the interstellar medium) and ours (integral of SFR), we would estimate that about $40 \%$ and $70 \%$ of satellites, respectively, in our low and high stellar mass bins should have been mass quenched. This is consistent with what we observe at large radii in the low-mass bin, but, in the high-mass bin, the ZENS $f_{Q}$ at $R>R_{\text {vir }}$ is lower than this. However, at these high masses, the number of galaxies at $R>R_{\text {vir }}$ is small (see Figure 11) and the corresponding statistical uncertainty quite large. We therefore set the mass-quenched $f_{Q}$ value in the high-mass bin to the Peng et al. (2010) expectation of 0.7 , which is less than $2 \sigma$ discrepant from our observed value at $R>R_{\mathrm{vir}}$. We stress that this choice for the mass-quenched $f_{Q}$ value is not critical to our discussion below. The adopted mass-quenched $f_{Q}$ values are shown as horizontal red lines in the top-right panels of Figures 1(a) and (b).

Several physical mechanisms have been suggested to be the origin of mass quenching and of environment quenching. Independent of the physics behind each of these quenching channels, however, the observed increase in $f_{Q}$ with decreasing halo-centric radius in these figures must reflect the increased importance of satellite quenching toward the centers of the halos (we hatch the relevant areas in the top-right panels of Figures 1(a) and (b) to guide the eyes). If mass quenching and satellite quenching had a different ability to affect galaxy structure, then this would produce a clear trend with halocentric distance in our $B / T$-based morphological composition of the quenched satellite fractions shown in Figures 2(a) and (b): the morphology associated with mass quenching would dominate at large group radii, and that associated with satellite quenching would become increasingly more important (up to about a 50:50 split) toward the central group regions where the $f_{Q}$ has almost doubled. Put another way, since the radial variation of $f_{Q}$ is due to the variation in relative importance of the two quenching channels, we would expect, if these channels had different morphological signatures, to then see a variation of $f_{Q_{\mathrm{ETG}}}$ with $f_{Q}$. We see no such variation in $f_{Q_{\mathrm{ETG}}}$, and, as a result, we can reject this simple hypothesis. We conclude either that neither of the two quenching channels induces a morphological transformation, or that both of the two result in the same morphological changes.

Insights about the latter possibility can be obtained by comparing the morphological mix of the quenched satellites with that of the star-forming satellites at large radii, $R>R_{\mathrm{vir}}$, since these are presumably representative of the progenitors of the quenched population. The fraction of $R>R_{\mathrm{vir}}$ star-forming satellites that are morphological early types ${ }^{8}$ is substantially lower, in each mass bin, than the $f_{Q_{\mathrm{ETG}}}$ that we have found for the quenched satellites, i.e., $11 \%_{-0.03}^{+0.04}$ and $35 \%_{-0.13}^{+0.18}$ for the low and high stellar mass bins as compared with $50 \%$ and $65 \%$, respectively.

The difference in the early-type fractions of star-forming and quenched satellites may be taken to indicate that quenching is associated with structural changes in the galaxies, that is, changes affecting their underlying mass distribution, such as substantial growth of stellar mass in the bulge (or inner disk) components. It is well established that quenched galaxies have radial surface brightness profiles that are steeper than those of star-forming galaxies and are well described by high Sérsic

\footnotetext{
8 The fraction of star-forming satellites that have an early-type morphology is calculated in an analogous manner as done for $f_{O_{\mathrm{ETG}}}$, i.e., as the (completenesscorrected) fraction of star-forming, early-type satellites relative to the whole star-forming satellite population.
} 
Table 3

Results of the $z$ Test Performed on $f_{Q}$ and $f_{Q \mathrm{ETG}}$

$10^{10} \leqslant \log _{10}\left(M / M_{\odot}\right)<10^{10.7}$

$10^{10.7} \leqslant \log _{10}\left(M / M_{\odot}\right) \leqslant 10^{11.5}$

$z$ test for fractions at $M_{\text {Halo }} \in[12.8 / 13.2,13.7[$ and $[13.7,14.8]$

\begin{tabular}{|c|c|c|c|c|}
\hline & $p$ value $f_{Q}$ & $\begin{array}{c}p \\
\text { value } f_{Q_{\mathrm{ETG}}}\end{array}$ & $p$ value $f_{Q}$ & $\begin{array}{c}p \\
\text { value } f_{Q_{\mathrm{ETG}}}\end{array}$ \\
\hline All $R$ & 0.68 & 0.85 & 1.00 & 0.60 \\
\hline$R \leqslant 0.5 R_{200}$ & 0.69 & 1.00 & 0.21 & 1.00 \\
\hline $0.5<R \leqslant R_{200}$ & 0.78 & 0.74 & 1.00 & 1.00 \\
\hline$R>R_{200}$ & 0.13 & 0.09 & 0.11 & 0.81 \\
\hline
\end{tabular}

$z$ test for fractions at $R \leqslant 0.5 R_{200}$ and $R>R_{200}$

\begin{tabular}{|c|c|c|c|c|}
\hline \multirow{5}{*}{$\begin{array}{l}\text { All } M_{\text {Halo }} \\
{[12.2,13.2[} \\
{[12.8 / 13.2,13.7[} \\
{[13.7,14.8]}\end{array}$} & $p$ value $f_{Q}$ & $\stackrel{p}{p}$ value $f_{Q_{\mathrm{ETG}}}$ & $p$ value $f_{Q}$ & \multirow{2}{*}{$\begin{array}{c}p \\
\text { value } f_{Q_{\mathrm{ETG}}} \\
0.19\end{array}$} \\
\hline & $<0.01$ & 1.00 & $<0.01$ & \\
\hline & 1.00 & 0.73 & $\cdots$ & $\cdots$ \\
\hline & 0.06 & 0.56 & 1.00 & 0.29 \\
\hline & $<0.01$ & 0.15 & $<0.01$ & 1.00 \\
\hline & \multicolumn{4}{|c|}{$z$ test for fractions at $\delta_{\mathrm{LSS}} \in[-0.5,0.3 / 0.7[$ and $[0.7,2]$} \\
\hline & $p$ value $f_{Q}$ & $\begin{array}{c}p \\
\text { value } f_{Q_{\mathrm{ETG}}}\end{array}$ & $p$ value $f_{Q}$ & $\begin{array}{c}p \\
\text { value } f_{Q_{\mathrm{ETG}}}\end{array}$ \\
\hline All $R$ & 0.74 & 1.00 & 0.21 & 0.24 \\
\hline$R \leqslant 0.5 R_{200}$ & 0.80 & 0.64 & $\ldots$ & $\ldots$ \\
\hline $0.5<R \leqslant R_{200}$ & 0.60 & 1.00 & $\ldots$ & $\ldots$ \\
\hline$R>R_{200}$ & 1.00 & 0.44 & $\cdots$ & $\cdots$ \\
\hline$[12.2,13.2[$ & 0.02 & 0.79 & $\cdots$ & $\cdots$ \\
\hline$[13.2,13.7[$ & 0.63 & 0.77 & $\ldots$ & $\ldots$ \\
\hline
\end{tabular}

$z$ test for fractions at $\delta_{\mathrm{LSS}} \in[-0.5,0.3 / 0.7[$ and $[0.7,2]$

Note. Significance of the environmental dependence of $f_{Q}$ and $f_{Q_{\mathrm{ETG}}}$ on $R / R_{\text {vir }}, M_{\text {Halo }}$, and $\delta_{\text {LSS }}$. For each of the three environmental parameters, we provide the $p$ value (probability of obtaining the observed difference from the same parent sample) for a $z$ test performed on the fractions measured in the lowest and highest environmental bins (e.g., $R \leqslant 0.5 R_{200}$ versus $R>R_{200}$ or $M_{\text {Halo }} \in[12.8,13.2$ [ versus $[13.7,14.8]$ ). The galaxy samples are subdivided according to the other environmental indicators and galaxy mass, as in Tables 1-2 and Figures 1(a)-4(b). The $p$ values are calculated assuming a confidence level of $1 \%$. The double numbers in some of the halo mass to $\delta_{\text {LSS }}$ intervals reflect the different binning for low- and high-mass galaxies in Figures 1(a)-4(b).

indices (e.g., Kormendy et al. 2009 and references therein), reflecting high central mass densities (e.g., Fang et al. 2013 and references therein). Also, since the pioneering work of Mihos \& Hernquist (1994), inward flows of gaseous material have long been recognized to take place during galaxy mergers and through disk instabilities. Such inward gas flows are likely to contribute to the growth of stellar mass in galactic bulges (see, e.g., Courteau et al. 1996; MacArthur et al. 2003; Immeli et al. 2004; our own work, i.e., Carollo et al. 1997, 1998, 2001, 2007 and Carollo 1999, 2004; the comprehensive review by Kormendy \& Kennicutt 2004 and references therein; Bournaud et al. 2011, and many others). This idea has been further developed in more recent theoretical studies (e.g., Dekel \& Burkert 2014) and is suggested to be observed at high redshifts (e.g., Genzel et al. 2006; Cameron et al. 2011) and, at least for low-mass bulges, certainly observed in the local universe, where small bulges show signs of "rejuvenation" of their stellar populations, with up to $10 \%-30 \%$ of their total mass consistent with having formed in the past few gigayears (Thomas \& Davies 2006; Carollo et al. 2007). If mergers and disk instabilities were directly or indirectly connected with galactic quenching, it would not be implausible to draw a connection between quenching and the growth of bulges. Establishing the direction of causality in any such change would remain, however, very difficult. Quenching could be directly linked to the growth of the bulge (e.g., Martig et al. 2013; Genzel et al. 2014), or the quenching process itself could be associated with bulge growth, or the prominence of the bulge could be linked to a third property (e.g., galactic mass or the presence of an AGN) that itself controls the quenching of star formation.

A key but often neglected consideration is, however, that changes in the disk surface brightness profiles following quenching are almost certain to occur once star formation ceases, and this will inevitably increase the prominence of the bulges even if the bulge itself remains unchanged. The different early-type fractions in star-forming and quenched galaxies could therefore arise from changes in only the light distributions of galaxies, that is, in their light-defined $B / T$ values and thus morphologies, due in particular to differential surface brightness fading after star formation ceases. The effects of washing out the presence of massive bulges under highly starforming disks at $z \sim 2$ has been, for example, highlighted by Tacchella et al. (2015).

There are theoretical suggestions whereby neither mass nor environment quenching mechanisms would produce a structural change. For example, radio-mode AGN feedback can provide a quenching mechanism that leaves unaffected the mass distributions of bulges and disks (e.g., Gabor \& Davé 2012). Gas removal once the galaxies become satellites will also preserve disks. Since gas removal as a satellitequenching mechanism is implemented in several current traditional SAMs, this gives us the opportunity to quantitatively compare our results on $f_{Q}$ and $f_{Q_{\mathrm{ETG}}}$ with a couple of such SAMs in Section 5.4

In the next two sections we explore instead the global and the bulge and disk properties in the quenched and star-forming satellite populations. We study the effects of the fading of their disks (with no growth of bulges) to investigate whether structural as opposed to morphological changes are mainly responsible for the observed differences between these populations and how this can be connected with our results on the morphological fractions of mass- and environment-quenched galaxies.

\subsection{The Global Properties of Quenched and Star-forming Satellites and of Their Bulges and Disks}

We have argued in the previous section that the morphological outcome of the different quenching channels appears to be the same, even though these quenched satellites have different morphologies from the star-forming satellites, presumed to be representative of the progenitors of the quenched galaxies. Figure 5 shows in blue (upper panels) the distribution of the measured light-defined $B / T$ ratios for all star-forming satellites and in magenta (central panels) that in our original sample of quenched satellites $^{9}$ (we defer to the next section the

\footnotetext{
9 The distribution of measured light-defined $B / T$ ratios for the original sample of quenched satellites is here averaged over all environmental bins and, given the independence of $f_{Q_{\mathrm{ETC}}}$ on any environment, is very similar to the distributions corresponding to the individual points plotted in Figures 2(a) and (b).
} 

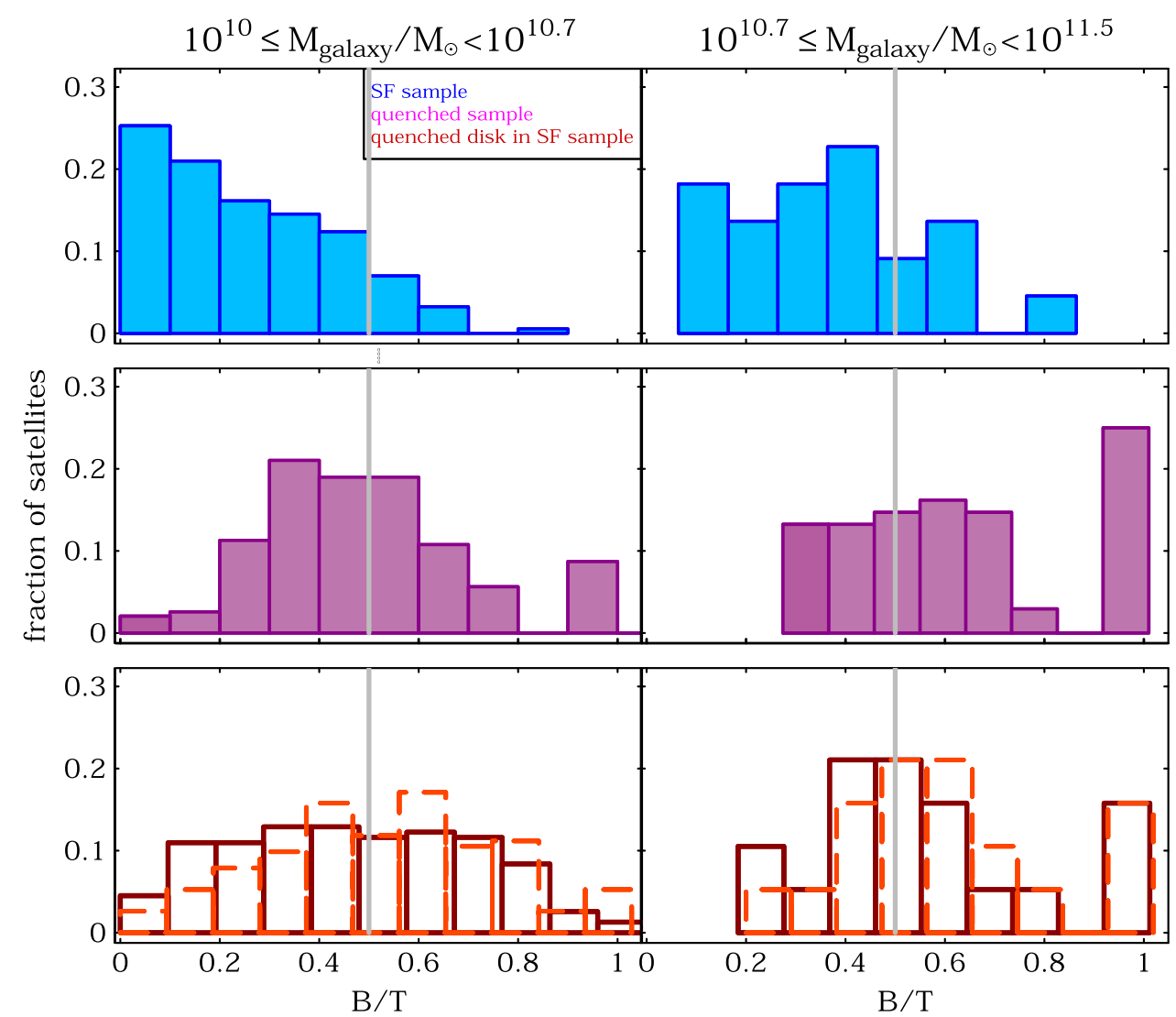

Figure 5. Distributions of measured, light-defined $B / T$ ratios in the $I$ band for ZENS satellites in the two galaxy mass bins of our study. The magenta histograms (central panels) correspond to the samples of quenched satellites that we study in this paper. Note that elliptical morphologies are placed at a value of $B / T=1$. The blue histograms (upper panels) show the distribution of $B / T$ for the original sample of ZENS star-forming satellites within the same bins of stellar mass. The red histograms (bottom panels) illustrate the effect on the measured $B / T$ of a (postquenching) radially uniform fading of the disk components of the star-forming satellite populations (dark red, solid $=1 \mathrm{Gyr}$ of passive evolution; light red, dashed $=3 \mathrm{Gyr}$ of passive evolution). The vertical gray lines in both mass bins show the $B / T$ threshold that we use to separate the two morphological classes of disk-dominated and early-type galaxies.

description of the lower panels of Figures 5 and 6). To reduce statistical errors, star-forming satellites at all halo-centric distances are included, but the following results do not change when using only the $R>R_{\mathrm{vir}}$ star-forming sample. The median $B / T$ values for the star-forming samples are $0.23_{-0.01}^{+0.02}$ and $0.37_{-0.05}^{+0.04}$ in the low and high stellar mass bins; the corresponding values for the quenched satellites are $0.47_{-0.02}^{+0.01}$ and $0.60_{-0.02}^{+0.05}$, respectively.

The quenched satellites also have smaller half-light radii than star-forming satellites. Figure 6 plots the histograms of total galaxy half-light radii $r_{1 / 2}$ for the star-forming (blue, upper panels) and the quenched (magenta, central panels) satellite samples: the average sizes of the quenched satellites are $2.7_{-0.1}^{+0.1} \mathrm{kpc}$ and $3.7_{-0.2}^{+0.2} \mathrm{kpc}$ at low and high stellar masses, compared with $4.8_{-0.2}^{+0.1} \mathrm{kpc}$ and $6.5_{-0.5}^{+1.0} \mathrm{kpc}$ for the star-forming samples. These values reflect the global mass-radius relations for quenched and star-forming nearby galaxies (see, e.g., the extensive discussion in Paper II and references therein).

The increased $B / T$ and the smaller half-light radii in the observed quenched satellites relative to the star-forming ones could be taken to indicate some growth of stellar mass in the bulges of star-forming satellites when they are moved from the star-forming "main sequence" (on the galaxy SFR versus $M_{\text {galaxy }}$ plane, e.g., Daddi et al. 2007) to the quenched population (either before quenching, i.e., in the gas flow regime, or after quenching, i.e., in the secular stellar disk bar- like instability regime; see Kormendy \& Kennicutt 2004 and references therein). In order to study this further, Figure 7 shows the surface brightness profiles for the bulge and disk components of the quenched and star-forming satellites that we derived from analytic, PSF-deconvolved bulge+disk decompositions in Paper II. In particular, we plot the typical profile of each component as a shaded area that encompasses the 25th and 75th percentiles of all surface brightness profiles. The average star-forming disk profile is shown in blue, and the average bulge in the star-forming satellites is shown in black. In magenta and gray we plot the observed disk and bulge profiles in the quenched satellites.

It is noticeable how similar the bulge profiles of star-forming and quenched satellites are at constant galaxy stellar mass, despite the substantially lower average light-based $B / T$ value measured for the former relative to the latter (but we discuss below a small effect in the inner profiles within $1 \mathrm{kpc}$; see insets in Figure 7). In contrast, the surface brightness profiles of the disks in the quenched satellites appear to be overall fainter and steeper (with smaller exponential scale lengths) than those of the presumed-progenitor star-forming disks of the same integrated galactic mass.

The fact that the overall bulge surface brightness profiles are so similar in the quenched and in the star-forming satellites argues in principle against substantial structural changes in the bulge components that are due to quenching. The two most stable parameters for the bulges are the total luminosities and 

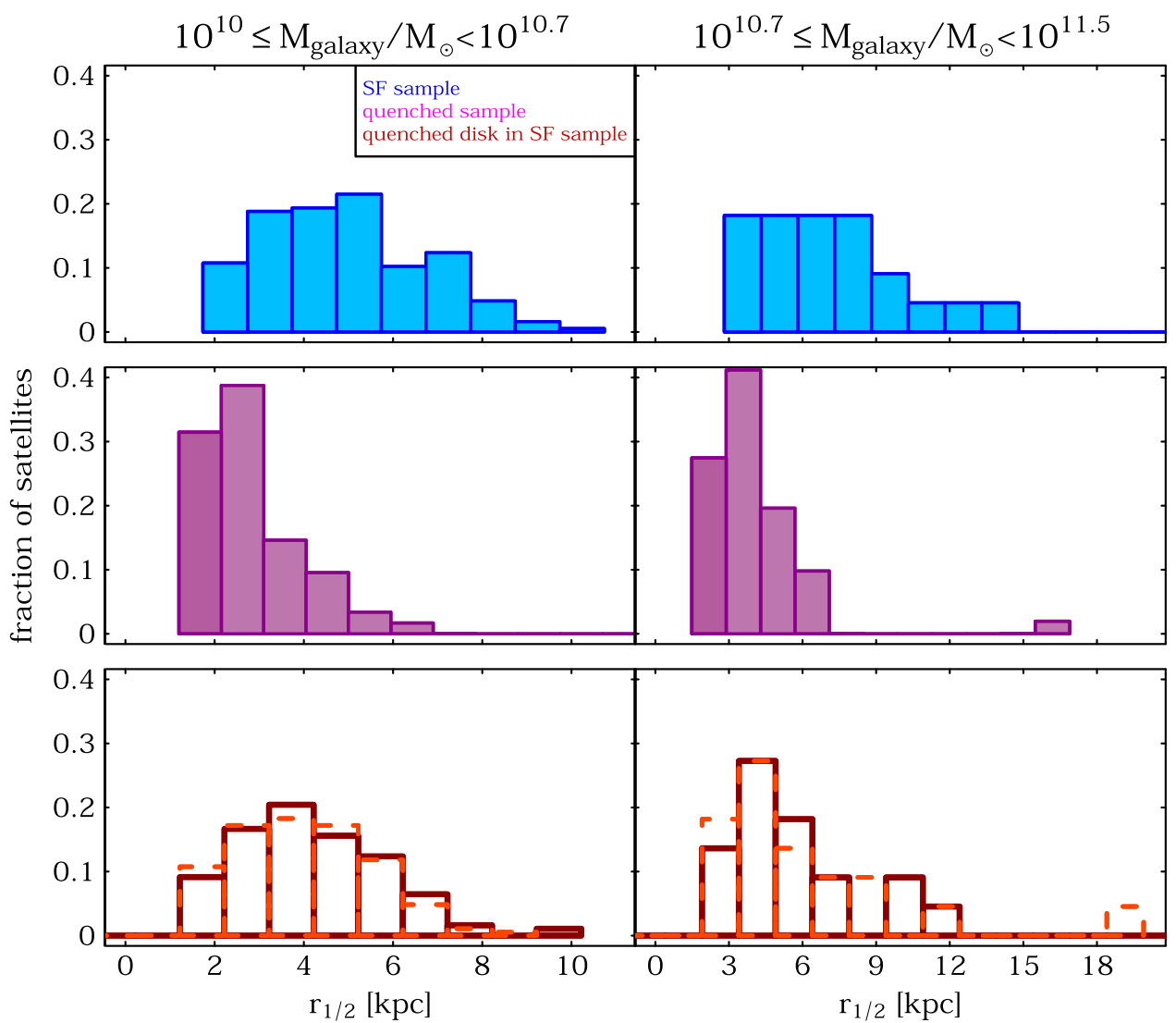

Figure 6. Distributions of galaxy half-light radii in the $I$ band for ZENS satellites in the two galaxy mass bins of our study. In magenta (central panels) we show the sample of quenched satellites that we study in this paper. In blue (upper panels) are the histograms for the original sample of ZENS star-forming satellites within the same bins of stellar mass. Similar to Figure 5, the red histograms (bottom panels) show the distributions of half-light radii measured for the artificially disk-faded satellite populations (using again as in Figure 5 a radially uniform fading model; dark red, solid $=1$ Gyr of passive evolution; light red, dashed $=3$ Gyr of passive evolution).

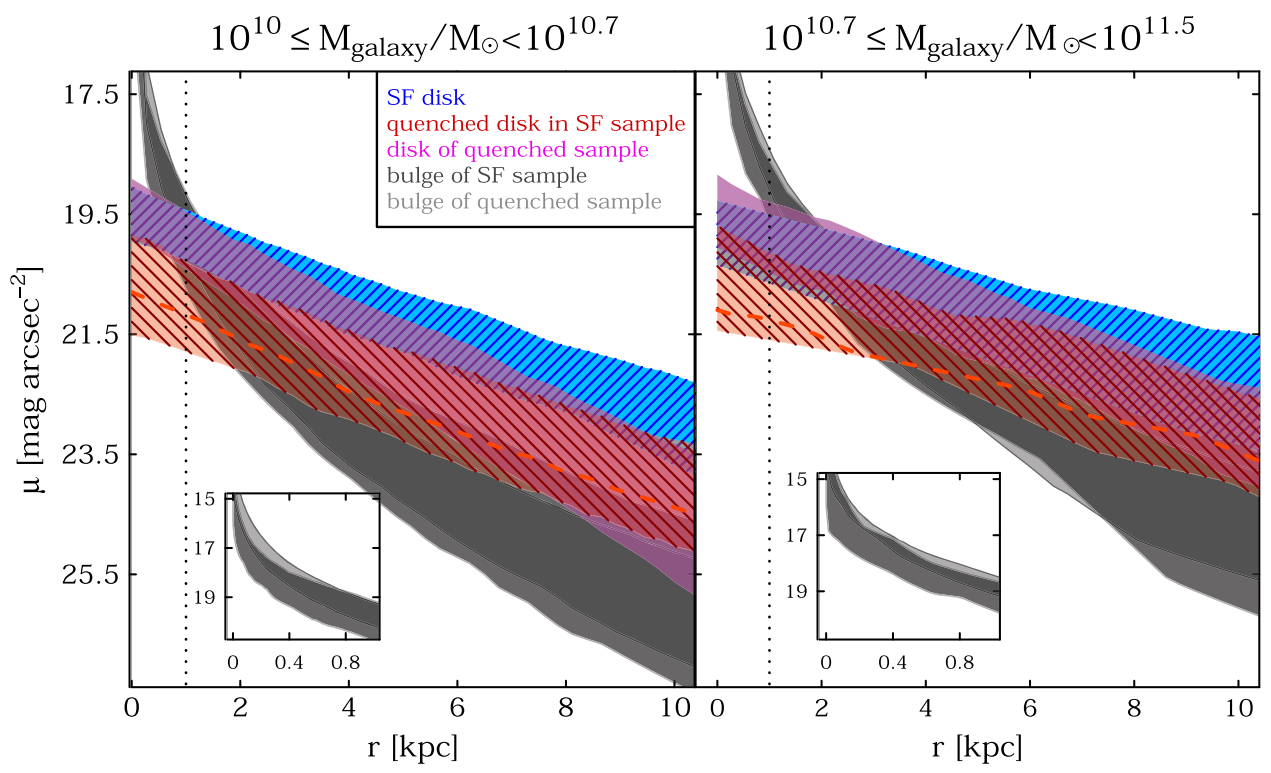

Figure 7. Average $I$-band surface brightness profiles for the bulges and disks in the sample of ZENS satellites in the two mass bins of our study (left and right). The shaded areas comprise the 25 th and 75 th percentiles of the surface brightness profile distributions for each galaxy component, and the profiles are obtained from the analytical model fits to the data, which are thus "PSF corrected." The typical size of the $I$-band PSF is indicated by the vertical dotted line. The bulges and disks of the quenched satellites are shown with the light gray and magenta curves, respectively. The corresponding components for the star-forming satellites are shown in dark gray and blue, respectively. With a red hatched area we show the artificially faded disks using a radially uniform fading model corresponding to 1 Gyr of passive evolution. The dashed orange line indicates the median surface brightness profiles obtained by applying instead a 3 Gyr of passive evolution (for clarity, the scatter for the disk-fading models is shown only for the $1 \mathrm{Gyr}$ passive evolution simulations but is similar for the $3 \mathrm{Gyr}$ model). The insets in both panels show zoomed-in radial profiles for the bulges of quenched and star-forming galaxies within the inner $1 \mathrm{kpc}$. 


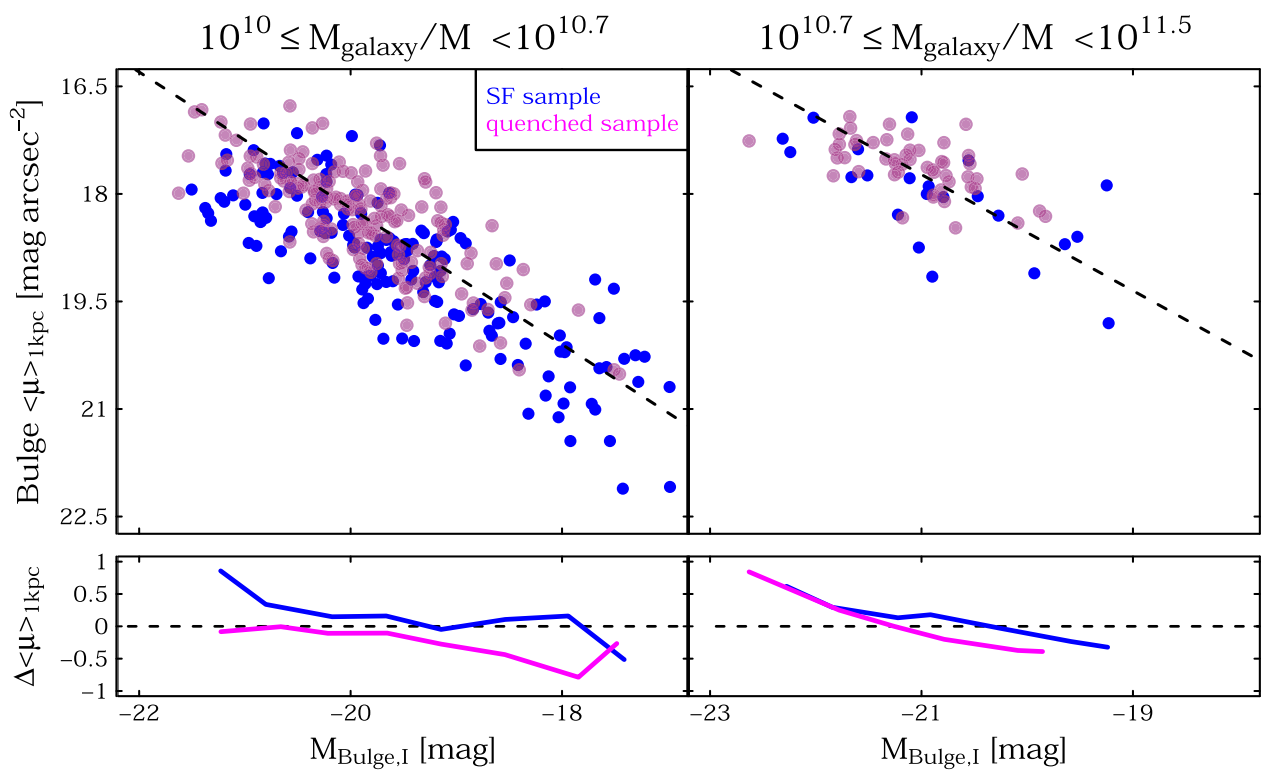

Figure 8. Top panels: the central bulge surface brightness within $1 \mathrm{kpc}$ (bulge $\left\langle\mu_{1 \mathrm{kpc}}\right\rangle$ ) plotted against the total $I$-band bulge absolute magnitude in the low (left) and high (right) galaxy mass bins (note that we use here magnitudes that are not $k$-corrected). As in the previous figures, the star-forming sample is plotted in blue and the quenched sample in magenta. The black dashed line shows the average relation for the total sample of quenched plus star-forming satellites. Bottom panels: the corresponding residuals relative to the average (black dashed lines) relations above for the quenched (magenta) and star-forming (blue) satellites.

the central surface brightnesses (see Paper II). This is also confirmed by the simulations that we describe in Section 5.3.

We therefore plot in Figure 8 the $I$-band bulge surface brightness within $1 \mathrm{kpc},\langle\mu\rangle_{1 \mathrm{kpc}}$, versus the total bulge $I$-band absolute magnitude for the quenched and star-forming satellites with a bulge component in the two bins of galaxy mass. The median of the total $I$-band bulge luminosities is similar in the star-forming and quenched satellites, that is, $I$-band absolute magnitudes of $-19.50_{-0.08}^{+0.11}$ (or $-19.82_{-0.06}^{+0.08}$ if we exclude galaxies with very small bulges, $B / T<0.1$, from the starforming sample) versus $-19.92_{-0.05}^{+0.05} \mathrm{mag}$ respectively in the low-mass bin, and $-20.94_{-0.15}^{+0.25}\left(-20.98_{-0.15}^{+0.15}\right)$ versus $-21.10_{-0.08}^{+0.08}$ at the high masses. There is a small shift in the average bulge surface brightness within $1 \mathrm{kpc}$ at constant I-band magnitude between the two satellite samples. This is better shown in the bottom panels of Figure 8, where we plot the residuals $\Delta\left\langle\mu_{1 \mathrm{kpc}}\right\rangle$ for the bulges in the quenched (magenta) and star-forming (blue) samples, relative to the average relation between bulge $\langle\mu\rangle_{1} \mathrm{kpc}$ and bulge $I$-band magnitude (indicated by the dashed black lines in the top panels of Figure 8). The linear parameters of these average fits in the low and high galaxy mass bins are respectively given by $a=0.95 \pm 0.03, b=37.2 \pm 0.6$ and $a=0.81 \pm 0.08, b=34.74 \pm 1.7$.

The small shift of order $0.3 \mathrm{mag}$ in bulge $\Delta\left\langle\mu_{1 \mathrm{kpc}}\right\rangle$ is consistent with a small amount of stellar mass being added to the centers of the bulges of quenched satellites. It is clear, however, that any such increase in the overall mass of the bulge cannot be substantial and in particular cannot significantly change the overall bulge (I-band) luminosity.

The comparison between the disk profiles of star-forming and quenched satellites, on the other hand, indicates that the change in the global light-defined $B / T$ and the reduction in the galaxy half-light radii between star-forming and quenched satellites are primarily associated with differences in their disks. Figure 9 shows the measured disk parameters for individual star-forming (blue) and quenched (magenta) satellites. These indicate that the central disk surface brightnesses averaged within the innermost $1 \mathrm{kpc}$ do not change very much $\left(\langle\mu\rangle_{\mathrm{SF}, 1 \mathrm{kpc}}=19.72_{-0.04}^{+0.05}\left[19.75_{-0.10}^{+0.16}\right] \mathrm{mag}\right.$ versus $\langle\mu\rangle_{Q, 1 \mathrm{kpc}}=19.74_{-0.05}^{+0.05}\left[19.59_{-0.09}^{+0.18}\right] \mathrm{mag}$ at low (high) galaxy masses), whereas the distribution of scale lengths of the disks in the quenched satellites is shifted to smaller values as compared with the scale lengths of star-forming satellites $\left(h_{\mathrm{SF}}=3.3_{-0.1}^{+0.1}\left[4.7_{-0.3}^{+0.7}\right] \mathrm{kpc}\right.$ versus $h_{Q}=2.2_{-0.1}^{+0.1}\left[3.3_{-0.2}^{+0.2}\right] \mathrm{kpc}$ again at low (high) masses).

We note that, because at least at late epochs (and possibly always) star formation is mostly associated with disks, a morphological change in the direction of an increase of the light-defined $B / T$ would be expected as an aftermath of quenching, even in the absence of structural (i.e., mass-based $B / T$ ) changes, simply due to the fading in surface brightness of a star-forming disk once star formation ceases. A fading of the disks would increase the apparent $B / T$ and shift galaxies toward earlier morphological types, potentially explaining the observed $B / T$ of the quenched satellites. Fading of a starforming disk in the aftermath of quenching clearly also reduces the overall half-light radius of a galaxy. This would also explain the smaller sizes of quenched satellites and has previously been invoked to help reconcile the differences between predicted and observed size functions of $\sim M *$ quenched galaxies at high redshifts in the context of the apparent change in size of the passive galaxy population (Carollo et al. 2013a). We therefore explore below in more detail the quantitative impact of disk fading on the global structure of galaxies.

\subsection{Morphological Changes Associated with the Fading of Disks in the Aftermath of Disk Quenching}

To quantify the impact of postquenching disk fading on the $B / T$ and sizes of galaxies, a first approach is simply to take the bulge-to-total ratio $B / T$ of the star-forming disks, $(B / T)_{\mathrm{SF}}$, and recompute the new "quenched" $(B / T)_{Q}$ that would be obtained 


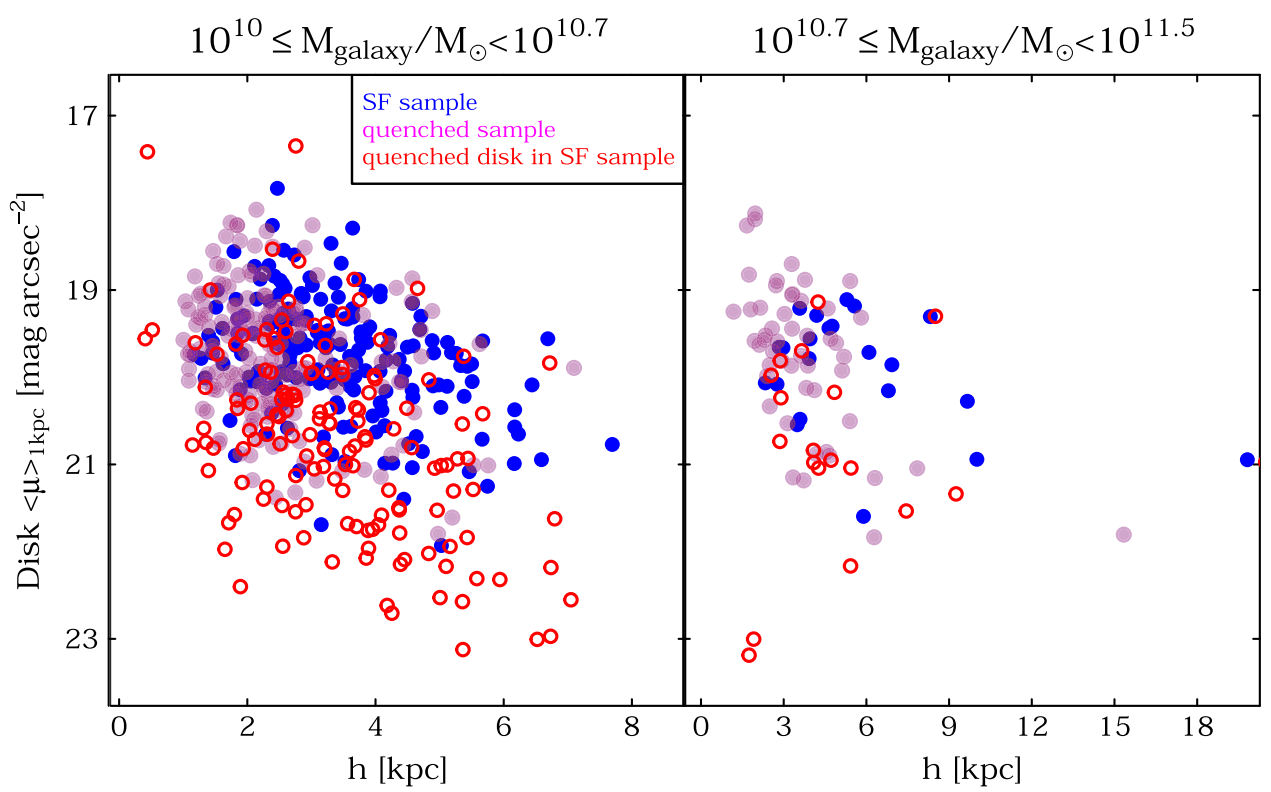

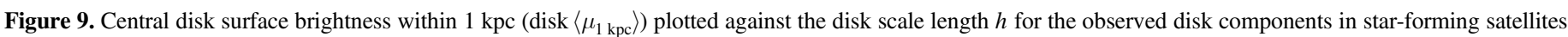

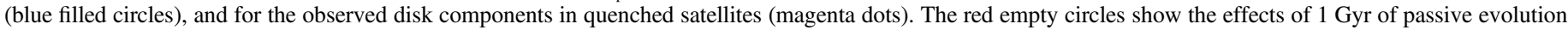
(postquenching) for the disk components of star-forming satellites.

with a reduced disk contribution. The resulting quenched $(B / T)_{Q}$ will be a nonlinear function of the initial $(B / T)_{\mathrm{SF}}$ and the reduced surface brightness of the quenched disk, which we represent by $\xi=D_{Q} / D_{\mathrm{SF}}<1$. Specifically, with

$$
(B / T)_{\mathrm{SF}}=\frac{B}{B+D_{\mathrm{SF}}},
$$

and under the assumption that disk fading is not accompanied by any change in the bulge component, $B$, we get

$$
(B / T)_{Q}=\frac{B}{B+D_{Q}}=\frac{(B / T)_{\mathrm{SF}}}{\xi+(1-\xi)(B / T)_{\mathrm{SF}}} .
$$

For a disk fading of about $1 \mathrm{mag}, \xi \sim 0.4$, a typical starforming galaxy with an original $(B / T)_{\mathrm{SF}}=0.4$ will have $(B / T)_{Q}=0.63$ after quenching, sufficient to make it an ETG.

We can look at this from a different perspective by looking at the actual surface brightness profiles of individual starforming galaxies in ZENS as decomposed into their bulge and disk components. Specifically, we use the light-defined $B / T$ decomposition for each star-forming satellite galaxy in ZENS (see Paper II and Figure 7), and we generate a simulated $I$-band image with the same bulge and disk structural parameters, except that the disk surface brightness is reduced, uniformly at all radii, by a certain amount: $\Delta \mu_{\text {fade }}=-2.5 \log \xi$. We consider fading of 1.0-1.5 mag, corresponding to the fading of a disk that has been forming stars at a constant rate prior to being quenched 1-3 Gyr before the current epoch of observation (using for this estimate the Bruzual \& Charlot 2003 stellar population synthesis models with a Chabrier 2003b initial mass

\footnotetext{
${ }^{10}$ As mentioned above, we also used these simulations to test which bulge and disk parameters can be considered as reliable. As also discussed in Paper II, the total magnitudes, scale lengths, and central surface brightnesses (within $1 \mathrm{kpc}$ ) of the disks, as well as the bulge total magnitudes and central surface brightnesses, are well recovered even after disk fading. In contrast, the bulge Sérsic indices and half-light radii are highly degenerate and unstable. We thus limit the comparisons between bulges and disks of different satellite samples to only those parameters that can be reliably measured.
}

function and a solar metallicity). These artificially quenched images are then convolved with the typical ZENS I-band PSF, and an appropriate level of noise is added. We then carry out the same bulge+disk decomposition fits as done for the real ZENS galaxies (see Section 2.4 and Paper II for details). The results ${ }^{10}$ are shown in red in Figures 5-7 and 9.

It is clear from Figures 5 and 7 that fading of the disk enhances the prominence of the central bulge, leading to a higher light-defined $B / T$. The light-defined $B / T$ values for the average galaxy profiles change at $10^{10}<M_{\odot}<10^{10.7} M_{\odot}$ from 0.21 for the star-forming average profile to 0.43 or 0.50 for the two fading models being examined; the corresponding change at $10^{10}<M_{\odot}<10^{10.7} M_{\odot}$ is from 0.35 to 0.57 or 0.64 . The median $B / T$ values of the distributions of individual $B / T$ shown in Figure 5 change from $0.23_{-0.01}^{+0.02}$ and $0.37_{-0.05}^{+0.04}$ for the original star-forming galaxies in the low and high stellar mass bins, to an average of about $0.50 \pm 0.02$ and $0.55 \pm 0.06$ for both low and high masses for the 1 Gyr (dark red solid) and 3 Gyr (light red dashed) disk-fading models, respectively. These values are quite close to the $0.47_{-0.02}^{+0.01}$ and $0.60_{-0.02}^{+0.05}$ medians of the $B / T$ distributions of real quenched satellites in the two mass bins. A Mann-Whitney-Wilcoxon $U$ test (or a Kolmogorov-Smirnov test) indicates that the probability that the measured $B / T$ distributions of the quenched satellites and the 1-Gyr-old artificially faded star-forming satellites are drawn from the same parent samples are substantial (precisely, $p$ values $>55 \%$ or $>6 \%$ and $>10 \%$ or $>30 \%$ in the low- and high-mass bins for the two tests, respectively; the corresponding values for a $t$ test are $>25 \%$ and $>10 \%$ ).

Figure 6 shows, however, that the galaxy half-light radii $r_{1 / 2}$ obtained from these radially uniform fading models (e.g., $4.1_{-0.1}^{+0.1} \mathrm{kpc}$ and $5.4_{-0.4}^{+1.0} \mathrm{kpc}$ in the two mass bins using the representative $1 \mathrm{Gyr}$ postquenching model) remain still significantly larger than those of the actual quenched satellites, $2.7 \mathrm{kpc}$ and $3.7 \mathrm{kpc}$, respectively. The disk scale lengths of the artificially faded disks are also too large $\left(3.2_{-0.1}^{+0.1} \mathrm{kpc}\right.$ and $4.3_{-0.3}^{+0.7} \mathrm{kpc}$ at low and high masses, respectively), and their 
central surface brightnesses are too faint $\left(20.68_{-0.08}^{+0.08}\right.$ and $20.95_{-0.25}^{+0.14}$ mag; see Figure 9).

A reduction in scale length would, however, be expected, due to a differential fading of the disks with galactic radius, e.g., due to a stellar age gradient within the disks. Smaller sizes could also arise if disks were smaller, at given mass, at earlier epochs, when the quenched satellites were actually quenched. Both would be a natural consequence of an inside-out growth of individual disks. We can quantify the first effect (differential fading) as follows. We assume that both the original (starforming) and the subsequently quenched disks can be represented by exponential light profiles with respective scale lengths $h_{\mathrm{SF}}$ and $h_{Q}$ and central surface brightnesses $\mu_{S F, 0}$ and $\mu_{Q, 0}$. The radial change in surface brightness will itself be an exponential with scale length:

$$
h_{\text {fade }}=\frac{h_{\mathrm{SF}} h_{Q}}{h_{\mathrm{SF}}-h_{Q}}=\left(h_{Q}^{-1}-h_{\mathrm{SF}}^{-1}\right)^{-1} \text {. }
$$

With

$$
\mu_{\mathrm{SF}}(r)=\mu_{\mathrm{SF}, 0}+1.085 r h_{\mathrm{SF}}^{-1}
$$

and

$$
\mu_{Q}(r)=\mu_{Q, 0}+1.085 r h_{Q}^{-1},
$$

the fading $\Delta \mu(r)_{\text {fade }}$ at different radii will be given by

$$
\Delta \mu(r)_{\text {fade }}=\left(\mu_{Q, 0}-\mu_{S F, 0}\right)+1.085 r\left(h_{Q}^{-1}-h_{\mathrm{SF}}^{-1}\right) .
$$

An apparent halving of the scale length, i.e., $h_{Q} \sim 0.5 h_{\mathrm{SF}}$ (roughly what we see in our data) corresponds therefore to the application of a differential fading of the same scale length as the original disk, $h_{\text {fade }} \sim h_{\mathrm{SF}}$, and to $\Delta \mu(r)_{\text {fade }}=1.085 r h_{\mathrm{SF}}^{-1}$. Such little fading at the center, i.e., $\mu_{Q, 0}-\mu_{S F, 0} \sim 0$ and $1 \mathrm{mag}$ of fading at the original scale length of the star-forming disk (consistent with the 1 Gyr passive evolution model above), can thus completely explain the smaller sizes of quenched satellites relative to disk-faded star-forming satellites.

The overall conclusion from these analyses is that a simple fading of the disks after star formation ceases can, in principle, explain most of the change in the observed (light-defined) $B / T$ and in the mean half-light radii that is seen relative to a plausible set of star-forming progenitors, without the need for any substantial mass growth or other changes in the bulge components. This therefore also supports the idea that neither mass quenching nor satellite quenching produces a significant structural change in the stellar mass distribution of satellite galaxies. Clearly, this conclusion is based on analysis of galaxies at the present epoch, albeit reflecting quenching that happened at earlier epochs. It therefore may or may not hold for quenching occurring at very much earlier times, such as at $z \sim 2$ when passive galaxies first appear in substantial numbers (e.g., Ilbert et al. 2013).

\subsection{SAM Predictions for the Environmental Dependence of the Quenched Satellite Fraction and Its Morphological Composition}

Generally speaking, a long-standing problem of SAMs remains the overprediction of the total number of quenched satellites (Bower et al. 2006; De Lucia et al. 2006; Font et al. 2008; Kimm et al. 2009). Even recent models that implement more accurate prescriptions for the removal of the satellites' gas reservoirs still predict too many galaxies in the green valley between the red sequence and the blue cloud (Font et al. 2008). This is evident especially in Figure 1(a), in the low galaxy mass bin of our ZENS analysis and, although in a much reduced form, also in Figure 1(b) in our high galaxy mass bin. In both figures, the dotted and dashed black lines show respectively, for the SAMs of De Lucia et al. 2006 (DL06) and Guo et al. 2011 (G11), ${ }^{11}$ the dependence of $f_{Q}$ on the group halo mass (left panels) and halo-centric distance (right panels).

The main improvements in the G11 model relative to the earlier DL06 prescription are a more accurate calculation of the ram pressure from the intragroup medium and a more gradual resulting stripping of gas from satellites as these move toward the inner group regions. While the gradual gas stripping narrows the gap between the data and theoretical predictions by lowering the quenched satellite fractions in the G11 relative to the DL06 SAMs, the G11 model still overpredicts the absolute number of quenched satellites, in particular in our low galaxy mass bin. Most likely the star-formation histories of low-mass galaxies at $M_{\text {galaxy }}<M *$ must be improved, and, at the same time, these galaxies should be prevented from losing their gas too easily.

At face value, the recipes implemented in both the DL06 and G11 models do reasonably well in predicting almost no dependence of the total (i.e., integrated over $R$ ) quenched satellite fraction $f_{Q}$ on group halo mass and the dependence of $f_{Q}$ on $\delta_{\text {LSS }}$. Also, both models predict some decrease in the quenched satellite fraction with increasing halo-centric distance. In the low galaxy mass bin, although the instantaneous gas stripping algorithm at work in the DL06 models leads to a somewhat milder decrease in $f_{Q}$ with mass than the gradual gas stripping algorithm in G11 (which gives satellites the time to establish a more significant halo-centric radial trend in the total quenched fraction), the amplitude of the radial decrease in $f_{Q}$ in our data is roughly consistent with both models. In $\mathrm{G} 11$, the timescales for establishing the radial trend of $f_{Q}$ with $R / R_{\text {vir }}$ are typically $\sim 4$ Gyrs, or a factor of $\sim 2$ longer than the estimates for $\tau_{\text {quench }}$ that are given by our analysis of the color properties of satellites in our Paper III and by several independent studies (Balogh et al. 2000; Wang et al. 2007; Weinmann et al. 2009; Feldmann et al. 2010, 2011; Rasmussen et al. 2012). Together with the large overprediction of quenched galaxies with a disk morphology that we discuss below, this suggests that gas removal from disk satellites orbiting within a common group halo is likely a faster but less universal process than what is currently implemented in the SAMs. This is also suggested by some numerical simulations of satellite evolution in group potentials (e.g., Feldmann et al. 2010). In the high galaxy mass bin, the G11 model reproduces the observed trend of $f_{Q}$ with $R / R_{\text {vir }}$ at distances $R<R_{\text {vir }}$, although it shows a rather flat relation at halo-centric distances $R>R_{\text {vir }}$; the DL06 model fails instead to predict the observed decrease of $f_{Q}$ with $R / R_{\mathrm{vir}}$ even within the virial radius. Given this somewhat better performance of G11 in reproducing all observed global trends of $f_{Q}$ in both stellar mass bins, we adopt this model as the fiducial one for the comparison of the observed trends of $f_{Q_{\mathrm{ETG}}}$ with $M_{\text {Halo }}$ and $R$ (and $\delta_{\text {LSS }}$ ).

G11 define as morphological the early-type galaxies with a bulge-to-total ratio $B / T>0.7$ in stellar mass; in ZENS we adopted a similar $B / T$ threshold to select our early-type sample, but measured in I-band light. In Figures 2(a), (b), and 4(a), (b)

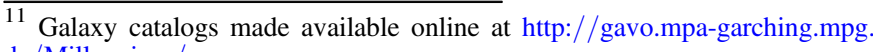
de/Millennium/.
} 
we overplot the model predictions for the $B / T>0.7$ sample as dash-dotted black lines. Given the difference in the definitions between data and models, we also plot equivalent model lines for a morphological mass selection equal to $B / T>0.5$ to explore the effects of the precise $B / T$ threshold value. The models are then treated as were the observations: for example, in the low galaxy mass bin (Figures 2(a) and 4(a)), where the number of galaxies enables a detailed investigation, the models are shown both integrated over the second environmental parameter (halo-centric distance for the plots with halo mass as the abscissa, and halo mass for the plots with halo-centric distance as the abscissa; top panels of the figures) and also split in the same bins of the second environmental parameter as done for the data (bottom panels).

The G11 model underpredicts the observed $f_{Q_{\mathrm{ETG}}}$. This discrepancy is larger in the low galaxy mass bin, where the amplitude of $f_{Q_{\mathrm{ETG}}}$ is underpredicted by a factor of $\sim 2-3$ (see Figures 2(a) and 7). At these masses, the dominant mechanism to form spheroids in the G11 model is via disk instabilities. These instabilities depend on internal galaxy properties only and not on environment (see Equation (34) in G11). Furthermore, the gradual environmental "strangulation" that is implemented in the G11 model leads to satellite quenching by the stripping of the hot gas halos while leaving their prequenching morphologies unaffected. ${ }^{12}$ This leaves the morphological mix of the quenched satellite fraction equal to that of the progenitor star-forming satellite population.

The main conclusions that we draw by comparing our data with the above SAMs, and in particular with the G11 model, is that in the regime of galaxy masses where environmentally driven satellite quenching starts being relevant, at galaxy masses below $M *$, the model (1) overpredicts the total quenched satellite fraction in all environments and (2) underpredicts the fraction of satellites with an early-type morphology in all environments: effectively it overpredicts the fraction of quenched satellites that retain a substantial disk component. This likely indicates that the model subjects too many starforming disk satellites to environmental quenching, thereby leading, at least at these galaxy mass scales, to too many quenched satellites with a disk-dominated morphology.

\section{SUMMARY AND CONCLUDING REMARKS}

Using the ZENS sample of nearby group halos in the mass range $M_{\text {Halo }} \sim 10^{12.2-14.9} M_{\odot}$, we have explored how, in the local universe, the fraction $f_{Q}$ of $10^{10-11.5} M_{\odot}$ satellites that are quenched and the morphological mix of these quenched satellites $f_{Q_{\mathrm{ETG}}}$ depend on group halo mass, on normalized halo-centric distance, and on the surrounding LSS density.

Similar to other studies, we find that the total quenched fraction $f_{Q}$ does not depend strongly on halo mass or on LSS density, with the exception of low-mass groups, which display an increase of $f_{Q}$ in the most extreme $\delta_{\text {LSS }}$ densities. At the galaxy mass scales that we probe, an average of about $50 \%$ (at low stellar masses with a median $\left.M_{\text {galaxy }} \sim 10^{10.35} M_{\odot}\right)$ and $\sim 70 \%$ (at high stellar masses with a median $M_{\text {galaxy }} \sim 10^{10.85} M_{\odot}$ ) of satellites have been quenched in one way or another. The fraction of quenched satellites increases with decreasing distance from the group centers, from $\lesssim 40 \%$ at

\footnotetext{
${ }^{12}$ Note that there is also tidal disruption of the stellar structures included in the G11 model, but when the condition for it to occur is satisfied, the whole galaxy is suddenly disrupted, which leads to it dropping out from the satellite sample.
}

$\sim 1.5$ virial radii up to $\gtrsim 60 \%$ in the group centers for satellites in the low galaxy mass bin, and $\sim 50 \%$ and $\gtrsim 80 \%$ for satellites in the high galaxy mass bin. These results are broadly consistent with previous findings, such as van den Bosch et al. (2008), Peng et al. (2012), and Wetzel et al. (2012).

The observed trends in $f_{Q}$ would be consistent with a picture of two independent quenching channels, one that acts on all galaxies and is independent of the environment of a galaxy (the mass-quenching channel of Peng et al. 2010), and another one that acts only on satellites and depends on halo-centric radius but not on halo mass (the environment or satellite quenching suggested by the same, and other, authors).

The morphological mix in the quenched satellite fraction, quantified by the fraction $f_{Q_{\mathrm{ETG}}}$ of quenched satellites that have an early-type morphology, remains on the other hand constant across all environments; that is, within errors we detect no significant variations in $f_{Q_{\mathrm{ETG}}}$ with halo mass in the range $\sim 10^{12.2 \rightarrow 14.9} M_{\odot}$, with halo-centric distance out to and beyond the virial radii of the group halos, or with LSS density (at least within the range probed by the ZENS group with masses $<10^{13.7} M_{\odot}$ ). At low and high galaxy stellar masses, about $50 \%$ and $70 \%$ of quenched satellites respectively have an early-type morphology in halos of all masses and at any halo-centric radius. The lack of any dependence on the halo-centric radius, despite the clear change in $f_{Q}$, shows that the morphological mix of quenched galaxies in our fixed-mass bins is decoupled from the overall quenched fraction.

The lack of any environmental trend with radius of the morphological mix of the quenched satellite population suggests that both mass and satellite quenching processes have the same transformational effect on the morphologies of the galaxies or that they have no effect at all. We furthermore note here that the idea of two independent quenching processes is argued in the 2010 Peng et al. analysis on the basis of the separability of their red fraction with mass and environment. It is, however, possible that the underlying physical mechanisms at work in satellite quenching are the same as for mass quenching, that is, that they are two different manifestations of the same underlying physical process. One way of doing this would be if satellite quenching is the result of a process linked to the parent halo mass. The mass independence of satellite quenching could then arise if the distribution of host halo masses for satellites (at the halo masses of interest) was largely independent of the stellar mass of the satellite, which would be supported by the independence of the satellite mass function on group halo mass (e.g., Peng et al. 2012).

Although there appears to be no difference in the morphological outcomes of the two quenching processes, the morphologies of the quenched satellites are, not surprisingly, systematically different from their star-forming counterparts. The quenched galaxies have higher $B / T$ and smaller half-light radii. The morphology-density relation of Dressler (1980) therefore appears to reflect changes in the quenched fraction with radius in the halo rather than changes in the morphological mix of quenched galaxies.

The total $I$-band luminosities and light profiles of the bulges of quenched satellites are very similar to those of the starforming ones. Any mass growth in the bulges associated with quenching cannot significantly change their luminosities. Instead, the changes in $B / T$ and in overall half-light radii are associated with differences in the disks. 
Surface brightness fading of previously star-forming disks after star formation ceases increases the morphological (lightdefined) $B / T$ and decreases the half-light radii, even if there are no underlying structural changes in the stellar mass distributions. We show that such fading can quantitatively explain the morphological differences in the $B / T$ between star-forming and quenched satellites and also will reduce the half-light radii. The quenched satellites also exhibit systematically smaller disk scale lengths, which further shrink the half-light radii relative to those given by uniform disk fading. These smaller disk scale lengths could be the result of either differential fading with radius in the disks, such as due to age gradients, or the disks of galaxies being systematically smaller at a given mass at earlier times. Both of these would be expected if individual disks grow inside-out.

The overall conclusion is that, at least at the relatively low redshifts being probed here, neither mass quenching nor satellite quenching is likely to change the mass-defined $B / T$, which is therefore probably set by other processes operating prior to the onset of quenching. This emerging picture from the low-redshift analysis is consistent with a merger origin for the most massive, pressure-supported spheroids in the local universe and with disk fading of bulge-dominated structures as a key process leading to the typical rotation-supported S0 +elliptical population around $M *$. Of course, the situation at much higher redshifts may or may not be the same.

Current SAMs postulate that early-type morphologies are the result of mergers preceding the infall of the satellite into the virialized group potential and that subsequent group halo physics, in the form of gradual removal of galaxies' hot gas halos, leaves the galaxy morphologies unchanged. This picture is not without challenges, not only because the models overpredict the total quenched satellite fraction in all environments, but also because the role of mergers and the quantitative merger rates need to be better defined as a function of cosmic epoch. Specifically, merger rates need to be made consistent with the emerging paradigm of a nonmerging, star-forming galaxy main sequence (e.g., Daddi et al. 2007), whose star formation is fed in a quasi-steady state from early times, by direct accretion of relatively cold gas streams (e.g., Dekel et al. 2009; see also Davé et al. 2012 and Lilly et al. 2013). Nevertheless, SAMs that include a progressive removal of the hot gas reservoir of galaxies within group halos can reproduce both the lack of dependence on halo mass and the dependence on halo-centric distance of the quenched satellite fraction that we see in our ZENS data. In all environments, they overpredict, however, the fraction of quenched satellites with a dominant disk component. Overall, the difficulty of the models in matching the observed quenched satellite population at redshift zero indicates that further model improvements should involve not only the physical recipes for gas removal from galaxies in heavy halos, but also their star-formation and mass-assembly histories as a function of galaxy mass.

CMC thanks Peter Capak, Nick Scoville, and the Caltech Astronomy Institute for the warm hospitality and the enjoyable and stimulating atmosphere during her sabbatical visit, when part of this paper was realised. We gratefully acknowledge support by the Swiss National Science Foundation. This publication makes use of data from ESO Large Program 177. A-0680. We also use data products from the Two Micron All Sky Survey, which is a joint project of the University of
Massachusetts and the Infrared Processing and Analysis Center/California Institute of Technology, funded by the National Aeronautics and Space Administration and the National Science Foundation. This research has made use of the NASA/IPAC Extragalactic Database (NED), which is operated by the Jet Propulsion Laboratory, California Institute of Technology, under contract with the National Aeronautics and Space Administration.

\section{APPENDIX \\ QUENCHED SATELLITE FRACTIONS AND THE MORPHOLOGIES OF QUENCHED SATELLITES IN RELAXED AND UNRELAXED GROUPS}

In ZENS about $40 \%$ of groups in the sample are defined as unrelaxed. As discussed in Section 2, this means it is not possible to identify a single galaxy with a stellar mass, location within the group, and velocity relative to the mean that can selfconsistently be ranked as the central galaxy in the group. In Paper I we argued that a substantial fraction of these unrelaxed groups can be the result of contamination from interloper galaxies, although about a quarter to a third of them are most likely genuinely young or merging groups.

To establish whether the groups defined as unrelaxed (regardless of the reason) have an impact on the fractions of quenched satellites in the different environments, we plot in Figure 10 the $R / R_{\text {vir }}$ versus $M_{\text {Halo }}$ plane for the two galaxy mass bins of our analysis. Each dot in these plots is a satellite galaxy: yellow dots are satellites in unrelaxed groups, and green dots indicate satellites in relaxed groups. Red circles around yellow and green symbols indicate that the given satellite is a quenched galaxy. The top half of Table 1 shows the values for the fractions $f_{Q}$ of satellites that are quenched in each of the bins in the $M_{\text {Halo }}$ versus $R / R_{\text {vir }}$ planes of Figure 10 that are delineated by the dotted lines. The table separately lists, for each galaxy stellar mass bin, the fractions $f_{Q}$ that are obtained when using the different group samples. The errors in Table 1 indicate Bayesian $\pm 1 \sigma$ confidence intervals for a binomial distribution (e.g., Cameron et al. 2011 and references therein). All fractions have been corrected for spectroscopic incompleteness in the parent $2 \mathrm{dFGRS}$ catalog. These corrections are small and are done by calculating the effective number of galaxies of a given category as the sum of the inverse galaxy weights $w_{i}$ defined in the appendix of Paper I and by characterizing the $2 \mathrm{dFGRS}$ completeness at the galaxy positions. For example, the effective number of quenched galaxies is equal to $N_{Q}=\sum_{i} 1 / w_{Q, i}$.

Figure 10 is repeated as Figure 11, but now the red circles indicate the quenched satellites that have an early-type morphology. Correspondingly, the bottom part of Table 1 reports the fractions $f_{Q_{\mathrm{ETG}}}$ of quenched satellites with an earlytype morphology, relative to the total population of quenched satellites in each given $R / R_{\mathrm{vir}}-M_{\text {Halo }}$ bin, again for the whole ZENS sample and for the sample of relaxed groups (and "clean relaxed" groups; see Section 2). Figures 12 and 13 show satellites in unrelaxed (yellow dots) and relaxed (green dots) groups, in different locations of the $R / R_{\text {vir }}$ versus $\delta_{\text {LSS }}$ plane, limiting the group sample to halo masses $M_{\text {Halo }} \leqslant 10^{13.7} M_{\odot}$ (for the reason discussed in Section 2). Correspondingly, for the low galaxy stellar mass bin, Table 2 lists the fractions of quenched satellites and the fraction of quenched satellites with an early-type morphology (relative to the total) in the bins of $\delta_{\text {LSS }}$ and $R / R_{\text {vir }}$ shown in the left panels of Figures 12 and 13 

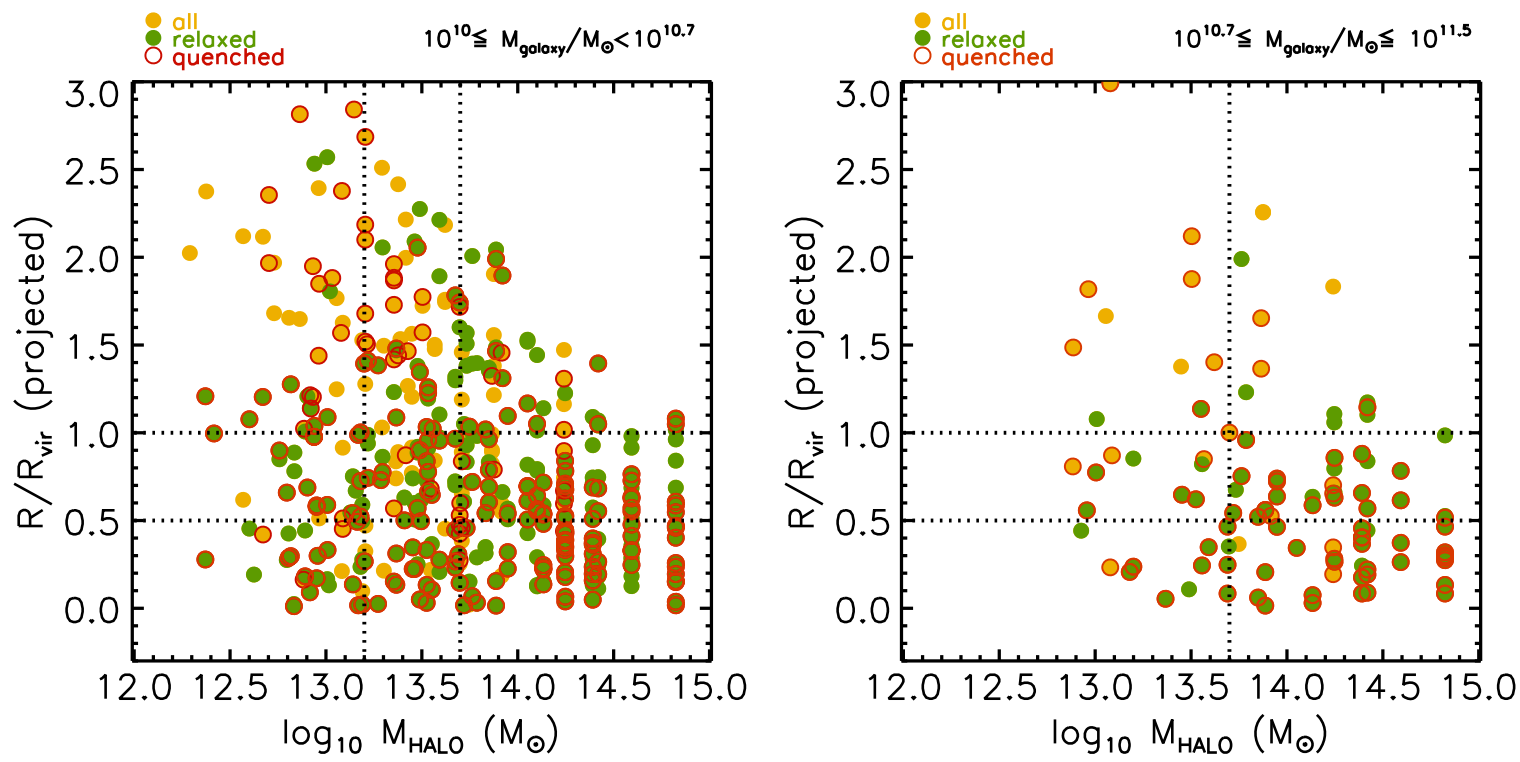

Figure 10. Left: satellite galaxies with stellar mass in the range $\log M \in[10,10.7]$ are plotted as dots in the halo-centric distance vs. halo mass plane. Yellow dots are satellites in ZENS groups that are classified as unrelaxed (see the text in the Appendix and Paper I), and green dots are those in dynamically relaxed groups. The surrounding red rings identify the quenched satellites, independent of their morphologies. Right: as in the left panel, but for satellites in the higher stellar mass bin $10^{10.7}-10^{11.5} M_{\odot}$.
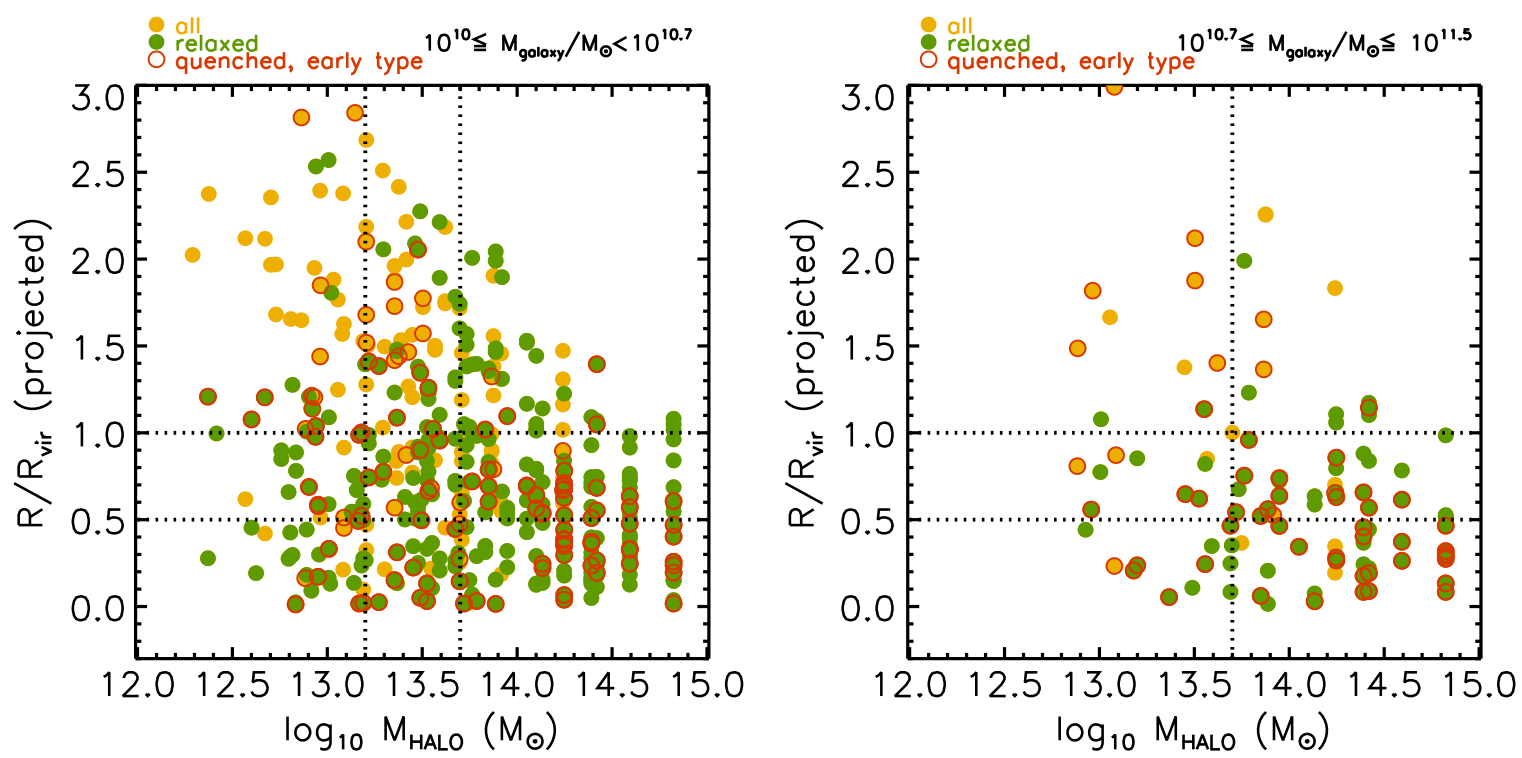

Figure 11. As in Figure 10, but now the red rings identify quenched satellites that have an early-type morphology.

(integrated over all $M_{\text {Halo }} \leqslant 10^{13.7} M_{\odot}$ groups). At high galaxy stellar masses, the ZENS $M_{\text {Halo }} \leqslant 10^{13.7} M_{\odot}$ group sample is too small to allow for a simultaneous split of the galaxy sample in both $\delta_{\mathrm{LSS}}$ and $R / R_{\mathrm{vir}}$. At these high galaxy stellar masses, we therefore only investigate in this paper the total fraction of quenched satellites and their morphologies in separate $\delta_{\text {LSS }}$ bins, by integrating not only over $M_{\text {Halo }} \leqslant 10^{13.7} M_{\odot}$ groups but also over all $R / R_{\text {vir }}$ values.

An inspection of Figures 10-13 shows that, at low halo masses and LSS densities, and in both galaxy stellar mass bins, it is the unrelaxed groups that are the dominant source of galaxies at large $R>R_{\mathrm{vir}}$ halo-centric distances. This is not surprising, given that such unrelaxed systems are either structures in the process of merging or unreliable physical associations with a misidentified central galaxy (and therefore group center). The nominal $R / R_{\text {vir }}$ values for galaxies in these systems are not therefore as meaningful as for the relaxed groups.

We note however that, as shown in Tables 1 and 2, the quenched fractions in most environmental bins are stable to the inclusion of the unrelaxed groups. The morphological compositions of the quenched satellite populations in the various environmental bins are also, within the errors, very similar, whether or not the unrelaxed groups are included in the analysis. The largest discrepancies are observed at high stellar masses; however, the statistical errors on the estimates make even these discrepancies only marginally significant.

We therefore conclude that both the quenched fraction of satellites and the morphological composition of this quenched population of satellites are largely independent of the implied dynamical state of the host group halos. In the analysis presented in this paper, we will thus consider the entire sample 

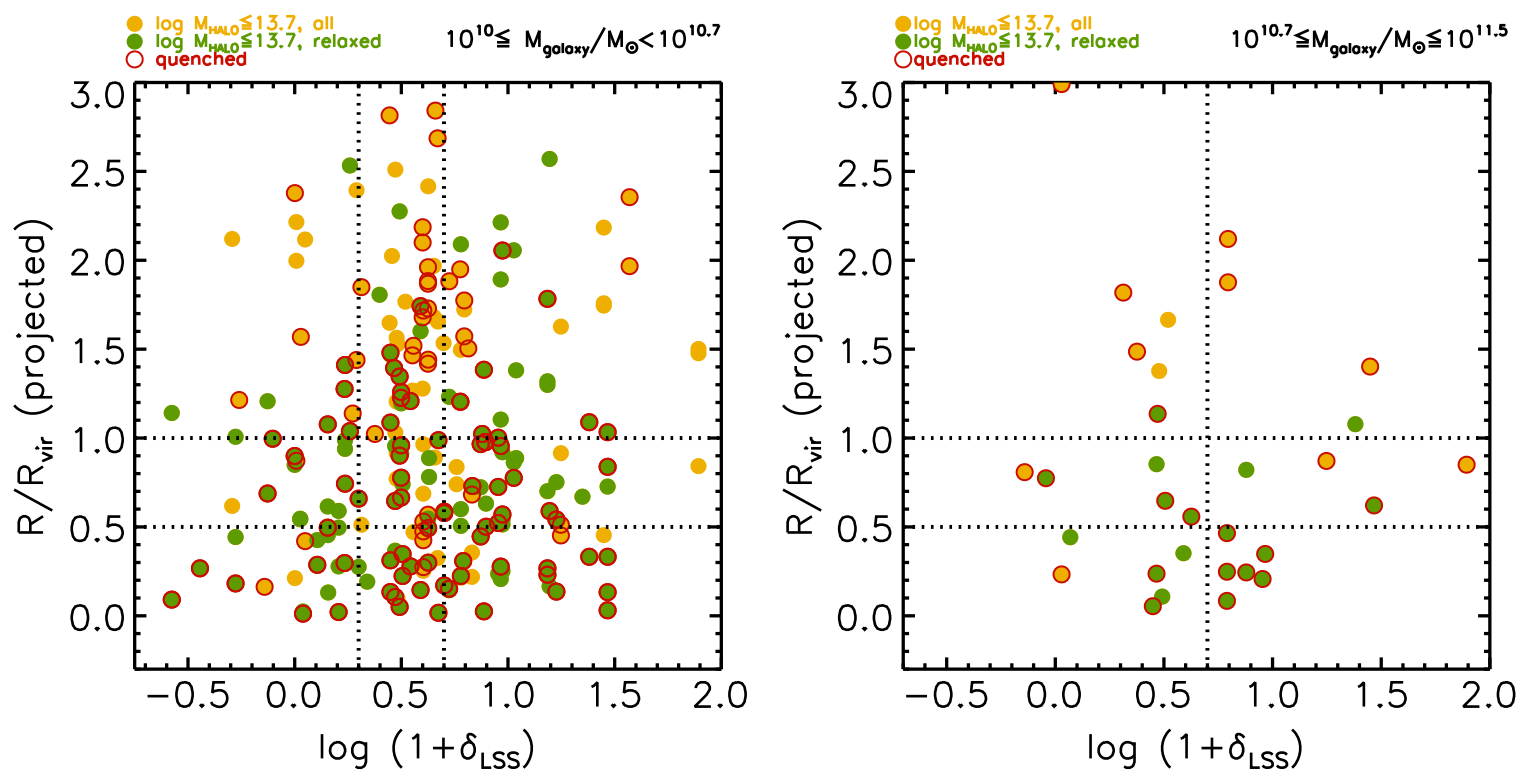

Figure 12. As in Figure 11, but now using the LSS overdensity as the environment parameter on the horizontal axis. Red rings identify quenched satellites, independent of their morphology.
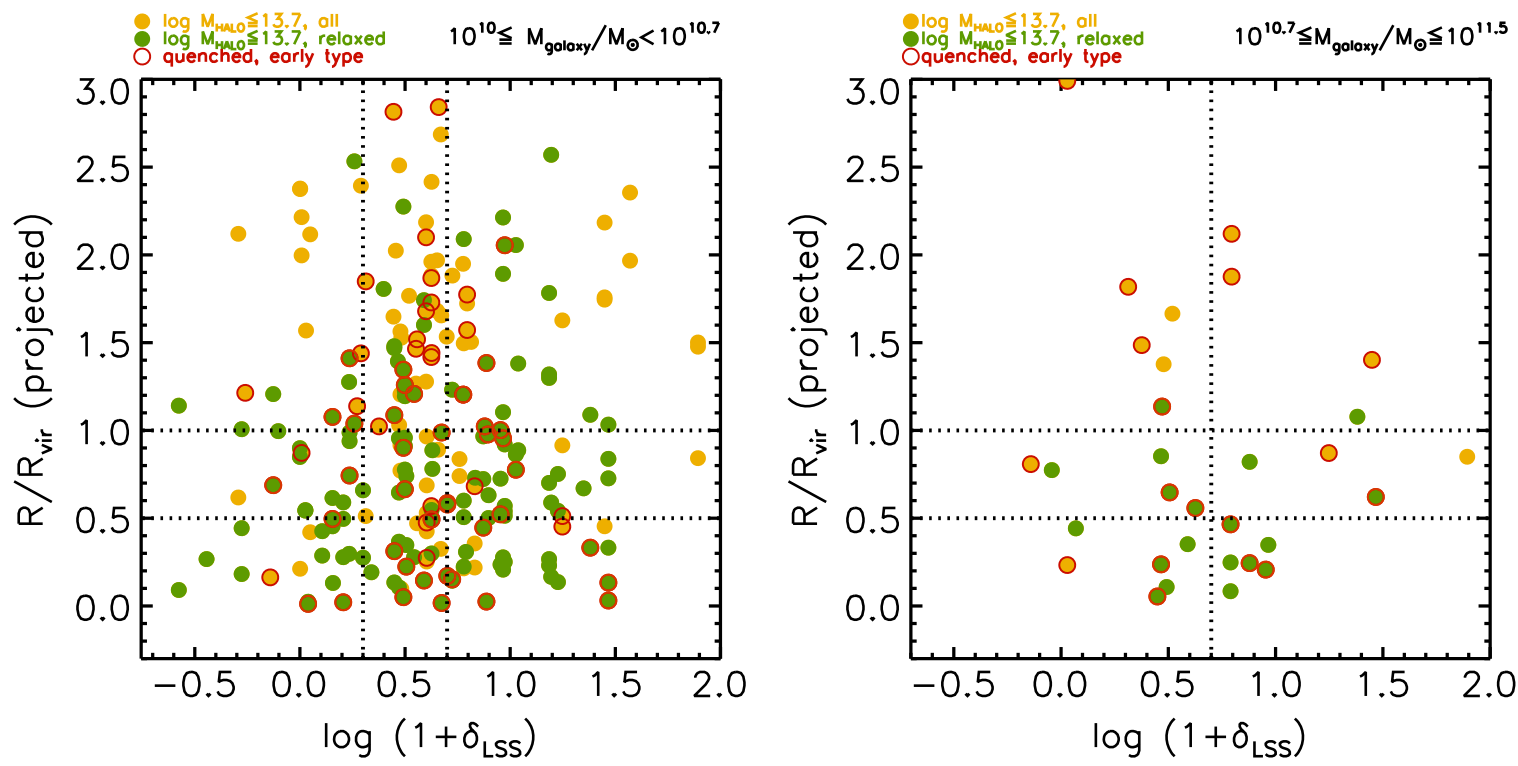

Figure 13. As in Figure 12, but with the red rings now indicating quenched galaxies with early-type morphology.

of relaxed plus unrelaxed ZENS groups so as to maximize the statistical significance of our results.

\section{REFERENCES}

Baldry, I. K., Balogh, M. L., Bower, R. G., et al. 2006, MNRAS, 373, 469 Balogh, M. L., Baldry, I. K., Nichol, R., et al. 2004, ApJL, 615, L101

Balogh, M. L., Navarro, J. F., \& Morris, S. L. 2000, ApJ, 540, 113

Birnboim, Y., \& Dekel, A. 2003, MNRAS, 345, 349

Birnboim, Y., Dekel, A., \& Neistein, E. 2007, MNRAS, 380, 339

Bournaud, F., Chapon, D., Teyssier, R., et al. 2011, ApJ, 730, 4

Bower, R. G., Benson, A. J., Malbon, R., et al. 2006, MNRAS, 370, 645

Bruzual, G., \& Charlot, S. 2003, MNRAS, 344, 1000

Bundy, K., Scarlata, C., Carollo, M., et al. 2010, ApJ, 719, 1969

Cameron, E., Carollo, C. M., Oesch, P. A., et al. 2011, ApJ, 743, 146

Carollo, C. M. 1999, ApJ, 523, 566

Carollo, C. M. 2004, in Coevolution of Black Holes and Galaxies, ed. L. C. Ho (Cambridge: Cambridge Univ. Press), 231

Carollo, C. M., Bschorr, T. J., Renzini, A., et al. 2013a, ApJ, 773, 112

Carollo, C. M., Cibinel, A., Lilly, S. J., et al. 2013b, ApJ, 776, 71 (Paper I)
Carollo, C. M., Scarlata, C., Stiavelli, M., Wyse, R. F. G., \& Mayer, L. 2007, ApJ, 658, 960

Carollo, C. M., Stiavelli, M., de Zeeuw, P. T., \& Mack, J. 1997, AJ, 114, 2366

Carollo, C. M., Stiavelli, M., de Zeeuw, P. T., Seigar, M., \& Dejonghe, H. 2001, ApJ, 546, 216

Carollo, C. M., Stiavelli, M., \& Mack, J. 1998, AJ, 116, 68

Cen, R. 2014, ApJ, 781, 38

Chabrier, G. 2003a, ApJL, 586, L133

Chabrier, G. 2003b, PASP, 115, 763

Cibinel, A., Carollo, C. M., Lilly, S. J., et al. 2013a, ApJ, 776, 72 (Paper II) Cibinel, A., Carollo, C. M., Lilly, S. J., et al. 2013b, ApJ, 777, 116 (Paper III) Colless, M., Dalton, G., Maddox, S., et al. 2001, MNRAS, 328, 1039

Conselice, C. J. 2003, ApJS, 147, 1

Courteau, S., de Jong, R. S., \& Broeils, A. H. 1996, ApJL, 457, L73

Daddi, E., Dickinson, M., Morrison, G., et al. 2007, ApJ, 670, 156

Davé, R., Finlator, K., \& Oppenheimer, B. D. 2012, MNRAS, 421, 98

De Lucia, G., Springel, V., White, S. D. M., Croton, D., \& Kauffmann, G. 2006, MNRAS, 366, 499

De Lucia, G., Weinmann, S., Poggianti, B. M., Aragón-Salamanca, A., \& Zaritsky, D. 2012, MNRAS, 423, 1277 
Dekel, A., \& Birnboim, Y. 2008, MNRAS, 383, 119

Dekel, A., \& Burkert, A. 2014, MNRAS, 438, 1870

Dekel, A., Sari, R., \& Ceverino, D. 2009, ApJ, 703, 785

Dressler, A. 1980, ApJ, 236, 351

Eke, V. R., Baugh, C. M., Cole, S., et al. 2004, MNRAS, 348, 866 Fang, J. J., Faber, S. M., Koo, D. C., \& Dekel, A. 2013, ApJ, 776, 63

Feldmann, R., Carollo, C. M., \& Mayer, L. 2011, ApJ, 736, 88

Feldmann, R., Carollo, C. M., Mayer, L., et al. 2010, ApJ, 709, 218

Feldmann, R., Carollo, C. M., Porciani, C., et al. 2006, MNRAS, 372, 565

Font, A. S., Bower, R. G., McCarthy, I. G., et al. 2008, MNRAS, 389, 1619

Gabor, J. M., \& Davé, R. 2012, MNRAS, 427, 1816

Gehrels, N. 1986, ApJ, 303, 336

Genzel, R., Förster Schreiber, N. M., Lang, P., et al. 2014, ApJ, 785, 75

Genzel, R., Tacconi, L. J., Eisenhauer, F., et al. 2006, Natur, 442, 786

George, M. R., Ma, C.-P., Bundy, K., et al. 2013, ApJ, 770, 113

Guo, Q., White, S., Boylan-Kolchin, M., et al. 2011, MNRAS, 413, 101

Haas, M. R., Schaye, J., Booth, C. M., et al. 2013, MNRAS, 435, 2931

Hearin, A. P., \& Watson, D. F. 2013, MNRAS, 435, 1313

Hoyle, B., Masters, K. L., Nichol, R. C., Jimenez, R., \& Bamford, S. P. 2012, MNRAS, 423, 3478

Ilbert, O., McCracken, H. J., Le Fèvre, O., et al. 2013, A\&A, 556, A55

Immeli, A., Samland, M., Gerhard, O., \& Westera, P. 2004, A\&A, 413, 547

Kartaltepe, J. S., Mozena, M., Kocevski, D., et al. 2015, ApJS, 221, 11

Kawata, D., \& Mulchaey, J. S. 2008, ApJL, 672, L103

Kimm, T., Somerville, R. S., Yi, S. K., et al. 2009, MNRAS, 394, 1131

Kormendy, J., Fisher, D. B., Cornell, M. E., \& Bender, R. 2009, ApJS, 182, 216

Kormendy, J., \& Kennicutt, R. C., Jr. 2004, ARA\&A, 42, 603

Kovač, K., Lilly, S. J., Knobel, C., et al. 2010, ApJ, 718, 86

Kovač, K., Lilly, S. J., Knobel, C., et al. 2014, MNRAS, 438, 717

Lilly, S. J., Carollo, C. M., Pipino, A., Renzini, A., \& Peng, Y. 2013, ApJ, 772, 119
Lilly, S. J., Le Brun, V., Maier, C., et al. 2009, ApJS, 184, 218

Lilly, S. J., Le Févre, O., Renzini, A., et al. 2007, ApJS, 172, 70

Lintott, C., Schawinski, K., Bamford, S., et al. 2011, MNRAS, 410, 166

Lotz, J. M., Primack, J., \& Madau, P. 2004, AJ, 128, 163

MacArthur, L. A., Courteau, S., \& Holtzman, J. A. 2003, ApJ, 582, 689

Martig, M., Bournaud, F., Teyssier, R., \& Dekel, A. 2009, ApJ, 707, 250

Martig, M., Crocker, A. F., Bournaud, F., et al. 2013, MNRAS, 432, 1914

Mihos, J. C., \& Hernquist, L. 1994, ApJL, 431, L9

Nair, P. B., \& Abraham, R. G. 2010, ApJS, 186, 427

Oesch, P. A., Carollo, C. M., Feldmen, R., et al. 2010, ApJL, 714, L47

Peng, Y.-j., Lilly, S. J., Kovač, R., et al. 2010, ApJ, 721, 193

Peng, Y.-j., Lilly, S. J., Renzini, A., \& Carollo, M. 2012, ApJ, 757, 4

Pipino, A., Cibinel, A., Tacchella, S., et al. 2014, ApJ, 797, 127

Rasmussen, J., Mulchaey, J. S., Bai, L., et al. 2012, ApJ, 757, 122

Schechter, P. 1976, ApJ, 203, 297

Tacchella, S., Carollo, C. M., Renzini, A., et al. 2015, Sci, 348, 314

Thomas, D., \& Davies, R. L. 2006, MNRAS, 366, 510

van den Bosch, F. C., Pasquali, A., Yang, X., et al. 2008, MNRAS, submitted (arXiv:0805.0002)

van der Wel, A. 2008, ApJL, 675, L13

Wang, L., Li, C., Kauffmann, G., \& De Lucia, G. 2007, MNRAS, 377, 1419

Weinmann, S. M., Kauffmann, G., van den Bosch, F. C., et al. 2009, MNRAS, 394,1213

Weinmann, S. M., van den Bosch, F. C., Yang, X., \& Mo, H. J. 2006, MNRAS, 366, 2

Wetzel, A. R., Tinker, J. L., \& Conroy, C. 2012, MNRAS, 424, 232

Wetzel, A. R., Tinker, J. L., Conroy, C., \& Bosch, F. C. v. d. 2014, MNRAS, 439, 2687

Woo, J., Dekel, A., Faber, S. M., et al. 2013, MNRAS, 428, 3306

York, D. G., Adelman, J., Anderson, J. E., Jr., et al. 2000, AJ, 120, 1579 